\title{
On Discontinuous and Continuous Approximations to Second-Kind Volterra Integral Equations
}

\author{
Hui Liang* \\ School of Science, Harbin Institute of Technology, Shenzhen, 518055, China
}

Received 13 August 2021; Accepted (in revised version) 16 September 2021

\begin{abstract}
Collocation and Galerkin methods in the discontinuous and globally continuous piecewise polynomial spaces, in short, denoted as DC, CC, DG and CG methods respectively, are employed to solve second-kind Volterra integral equations (VIEs). It is proved that the quadrature DG and CG (QDG and QCG) methods obtained from the DG and CG methods by approximating the inner products by suitable numerical quadrature formulas, are equivalent to the DC and CC methods, respectively. In addition, the fully discretised DG and CG (FDG and FCG) methods are equivalent to the corresponding fully discretised DC and CC (FDC and FCC) methods. The convergence theories are established for DG and CG methods, and their semi-discretised (QDG and QCG) and fully discretized (FDG and FCG) versions. In particular, it is proved that the CG method for second-kind VIEs possesses a similar convergence to the DG method for first-kind VIEs. Numerical examples illustrate the theoretical results.
\end{abstract}

AMS subject classifications: 45D05, 65R20

Key words: Volterra integral equations, collocation methods, Galerkin methods, discontinuous Galerkin methods, convergence analysis.

\section{Introduction}

In this paper, we consider the following Volterra integral equations (VIEs) of the second kind:

$$
u(t)=g(t)+\int_{0}^{t} K(t, s) u(s) d s, \quad t \in I:=[0, T]
$$

with continuous kernel, which arise widely as mathematical models of physical and biological phenomena (see the two monographs $[1,2]$ ). There are lots of researchers focus on the numerical methods of VIEs (see $[1,6-9,11-19,21]$ and the references cited

\footnotetext{
${ }^{*}$ Corresponding author. Email addresses: lianghui@hit.edu.cn; wise2peak@126.com (H. Liang) 
therein). Among these numerical methods, the collocation method is one of the most popular methods for (1.1). In general, collocation solutions are sought either in the space

$$
S_{m-1}^{(-1)}\left(I_{h}\right):=\left\{v:\left.v\right|_{\sigma_{n}} \in \mathcal{P}_{m-1}\left(\sigma_{n}\right)(0 \leq n \leq N-1)\right\}
$$

of discontinuous piecewise polynomials of degree $m-1 \geq 0$, or in the space of globally continuous piecewise polynomials of degree $m \geq 1$ (see $[1,13,14]$ )

$$
S_{m}^{(0)}\left(I_{h}\right):=\left\{v \in C(I):\left.v\right|_{\sigma_{n}} \in \mathcal{P}_{m}\left(\sigma_{n}\right)(0 \leq n \leq N-1)\right\} .
$$

Here,

$$
I_{h}:=\left\{t_{n}:=n h: n=0, \ldots, N\left(t_{N}:=T\right)\right\}
$$

denotes a given mesh on $I=[0, T]$, with mesh diameter $h:=T / N$, and $\mathcal{P}_{k}=\mathcal{P}_{k}\left(\sigma_{n}\right)$ is the linear space of (real) polynomials on $\sigma_{n}:=\left[t_{n}, t_{n+1}\right]$ of degree not exceeding $k$. For a given mesh $I_{h}$ the set

$$
X_{h}:=\left\{t_{n, i}:=t_{n}+c_{i} h: 0<c_{1}<\cdots<c_{m} \leq 1(0 \leq n \leq N-1)\right\}
$$

will denote the collocation points corresponding to prescribed collocation parameters $\left\{c_{i}\right\}$.

In [13], it is proved that the collocation solution in the globally continuous piecewise polynomial space $S_{m}^{(0)}\left(I_{h}\right)$ for the second-kind VIE (1.1) converges to the exact solution, if and only if the collocation parameters $\left\{c_{i}\right\}$ satisfy the following condition:

$$
-1 \leq l_{0}(1)=(-1)^{m} \prod_{i=1}^{m} \frac{1-c_{i}}{c_{i}} \leq 1,
$$

where $l_{0}(s)$ is defined in (3.3) of Section 3.1.1. It is very interesting that (1.2) is also the sufficient and necessary condition for the collocation solution in the discontinuous polynomial space $S_{m-1}^{(-1)}\left(I_{h}\right)$ converging to the exact solution of the following first-kind VIE (see [1, Theorem 2.4.2]):

$$
0=g(t)+\int_{0}^{t} K(t, s) u(s) d s, \quad t \in I:=[0, T]
$$

with $|K(t, t)| \geq k_{0}>0$. It means that the continuous collocation (CC) method in the globally continuous piecewise polynomial space $S_{m}^{(0)}\left(I_{h}\right)$ for the second-kind VIE (1.1) has a similar convergence to the discontinuous collocation (DC) method in the discontinuous piecewise polynomial space $S_{m-1}^{(-1)}\left(I_{h}\right)$ for the first-kind VIE (1.3).

In 2009, [3] investigated the discontinuous Galerkin (DG) method in the discontinuous piecewise polynomial space $S_{m-1}^{(-1)}\left(I_{h}\right)$ for the first-kind VIE (1.3), and it is proved that an $(m-1)$-th degree DG approximation exhibits global convergence of order $m-1$ when $m$ is even and order $m$ when $m$ is odd. A natural question is that does the continuous Galerkin (CG) method in the globally continuous piecewise polynomial space 
$S_{m}^{(0)}\left(I_{h}\right)$ for the second-kind VIE (1.1) possess a similar convergence? This is one of the core parts of this paper, and we will give an affirmative answer in Theorem 3.5 of Section 3.4.

Notice that the cardinality of $X_{h}$ is $\left|X_{h}\right|=N m$, and

$$
\operatorname{dim} S_{m-1}^{(-1)}\left(I_{h}\right)=N m \quad \text { and } \operatorname{dim} S_{m}^{(0)}\left(I_{h}\right)=N m+1,
$$

so for the VIE (1.1), the DC method in discontinuous polynomial collocation space $S_{m-1}^{(-1)}\left(I_{h}\right)$ is more natural than the CC method in globally continuous polynomial collocation space $S_{m}^{(0)}\left(I_{h}\right)$ (see $[13,14]$ ). Therefore for (1.1), in this paper, we will first investigate DG methods, and the relationship between DG and DC methods, then analyze CG methods, and the relationship between CG and CC methods.

This paper deals with the second-kind VIE (1.1), and the outline is mainly divided into two parts. Section 2 is the first part, and it mainly focuses on discontinuous approximations, including DC and DG methods: First, we will review the DC and fully discretised DC (FDC) schemes. Second, we will introduce the DG, quadrature DG (QDG) and fully discretised DG (FDG) schemes, then investigate the relationships between QDG and DC schemes, FDG and FDC schemes, respectively. Last, we will review the convergence for the DC method and give the detailed convergence analysis for the DG method. Section 3 is the second part, and it mainly focuses on continuous approximations, including CC and CG methods: the organization is as same as the first part, but now DC, FDC, DG, QDG and FDG are replaced as CC, FCC, CG, QCG and FCG. Finally, in Section 4, some numerical experiments are given to illustrate the theoretical results.

\section{Discontinuous approximations}

\subsection{Review: DC approximations}

In [1, Chapter 2], both DC and FDC methods are investigated thoroughly for VIE (1.1). In order to establish the relationship between DC and DG methods, in this subsection, we will review DC and FDC methods briefly.

\subsubsection{DC schemes}

Denote $u_{D C} \in S_{m-1}^{(-1)}\left(I_{h}\right)$ as the DC solution of (1.1), then the collocation equation is

$$
u_{D C}(t)=g(t)+\int_{0}^{t} K(t, s) u_{D C}(s) d s, \quad t \in X_{h} .
$$

Since $u_{D C} \in S_{m-1}^{(-1)}\left(I_{h}\right)$, the local representation of the collocation solution $u_{D C}$ on the subinterval $\sigma_{n}$ can be written as

$$
u_{D C}\left(t_{n}+s h\right)=\sum_{j=1}^{m} L_{j}(s)\left(U_{D C}^{n}\right)_{j}, \quad s \in(0,1],
$$


where $\left(U_{D C}^{n}\right)_{j}:=u_{D C}\left(t_{n, j}\right)$, and

$$
L_{j}(s):=\prod_{k=1, k \neq j}^{m} \frac{s-c_{k}}{c_{j}-c_{k}}, \quad j=1, \ldots, m
$$

denote the Lagrange basis functions with respect to the distinct points $\left\{c_{i}\right\}$. Therefore, by the collocation equation (2.1) and the local representation (2.2), we obtain

$$
\begin{aligned}
u_{D C}\left(t_{n, i}\right)= & g\left(t_{n, i}\right)+\int_{0}^{t_{n, i}} K\left(t_{n, i}, s\right) u_{D C}(s) d s \\
= & g\left(t_{n, i}\right)+h \int_{0}^{c_{i}} K\left(t_{n, i}, t_{n}+s h\right) u_{D C}\left(t_{n}+s h\right) d s \\
& +h \sum_{l=0}^{n-1} \int_{0}^{1} K\left(t_{n, i}, t_{l}+s h\right) u_{D C}\left(t_{l}+s h\right) d s \\
= & g\left(t_{n, i}\right)+h \sum_{j=1}^{m} \int_{0}^{c_{i}} K\left(t_{n, i}, t_{n}+s h\right) L_{j}(s) d s\left(U_{D C}^{n}\right)_{j} \\
& +h \sum_{l=0}^{n-1} \sum_{j=1}^{m} \int_{0}^{1} K\left(t_{n, i}, t_{l}+s h\right) L_{j}(s) d s\left(U_{D C}^{l}\right)_{j} .
\end{aligned}
$$

Denote

$$
\begin{aligned}
& \mathbf{G}_{D C}^{n}:=\left(g\left(t_{n, 1}\right), \ldots, g\left(t_{n, m}\right)\right)^{T}, \quad \mathbf{U}_{D C}^{n}:=\left(\left(U_{D C}^{n}\right)_{1}, \ldots,\left(U_{D C}^{n}\right)_{m}\right)^{T}, \\
& \mathbf{B}_{D C}^{n}:=\left(\begin{array}{c}
\int_{0}^{c_{i}} K\left(t_{n, i}, t_{n}+s h\right) L_{j}(s) d s \\
(i, j=1, \ldots, m)
\end{array}\right), \\
& \mathbf{B}_{D C}^{(n, l)}:=\left(\begin{array}{c}
\int_{0}^{1} K\left(t_{n, i}, t_{l}+s h\right) L_{j}(s) d s \\
(i, j=1, \ldots, m)
\end{array}\right) \quad(0 \leq l<n \leq N-1) .
\end{aligned}
$$

Then the collocation equation can be written as the following concise form:

$$
\left(\mathbf{I}_{m}-h \mathbf{B}_{D C}^{n}\right) \mathbf{U}_{D C}^{n}=\mathbf{G}_{D C}^{n}+h \sum_{l=0}^{n-1} \mathbf{B}_{D C}^{(n, l)} \mathbf{U}_{D C}^{l},
$$

where $\mathbf{I}_{m}$ denotes the identity matrix in $\mathbb{R}^{m \times m}$.

If $g \in C(I)$ and $K \in C(D)$, where $D:=\{(t, s): 0 \leq s \leq t \leq T\}$, then the matrix $\mathbf{B}_{D C}^{n}$ is bounded, so by Neumann Lemma, there exists a constant $h_{D C}>0$ such that for any $h \in\left(0, h_{D C}\right)$, the linear algebraic system (2.4) has a unique solution $\mathbf{U}_{D C}^{n}$ for all $n=0, \ldots, N-1$. Hence (2.1) determines a unique DC solution $u_{D C} \in S_{m-1}^{(-1)}\left(I_{h}\right)$ for (1.1) whose local representation on $\sigma_{n}$ is given by (2.2). 


\subsubsection{FDC schemes}

In general, the integrals of $\mathbf{B}_{D C}^{n}, \mathbf{B}_{D C}^{(n, l)}$ in (2.4) cannot be found analytically, but have to be approximated by suitable numerical quadrature formulas, and the resulting scheme is the so-called fully discretised DC (FDC) scheme.

Let $\hat{u}_{D C} \in S_{m-1}^{(-1)}\left(I_{h}\right)$ be the FDC solution. Then the local representation of $\hat{u}_{D C}$ on the subinterval $\sigma_{n}$ can be written as

$$
\hat{u}_{D C}\left(t_{n}+s h\right)=\sum_{j=1}^{m} L_{j}(s)\left(\hat{U}_{D C}^{n}\right)_{j}, \quad s \in(0,1],
$$

where $\left(\hat{U}_{D C}^{n}\right)_{j}:=\hat{u}_{D C}\left(t_{n, j}\right)$.

On $\sigma_{n}$, we choose interpolatory $m$-point quadrature formulas whose abscissas are based on the $m$ collocation parameters $\left\{c_{i}\right\}$, and denote

$$
b_{i}:=\int_{0}^{1} L_{i}(s) d s
$$

as the corresponding weights. Then by (2.3), we have

$$
\begin{aligned}
\left(\hat{U}_{D C}^{n}\right)_{i}= & g\left(t_{n, i}\right)+h c_{i} \sum_{j=1}^{m} \sum_{k=1}^{m} K\left(t_{n, i}, t_{n}+c_{i} c_{k} h\right) L_{j}\left(c_{i} c_{k}\right) b_{k}\left(\hat{U}_{D C}^{n}\right)_{j} \\
& +h \sum_{l=0}^{n-1} \sum_{j=1}^{m} \sum_{k=1}^{m} K\left(t_{n, i}, t_{l, k}\right) L_{j}\left(c_{k}\right) b_{k}\left(\hat{U}_{D C}^{l}\right)_{j} \\
= & g\left(t_{n, i}\right)+h c_{i} \sum_{j=1}^{m} \sum_{k=1}^{m} K\left(t_{n, i}, t_{n}+c_{i} c_{k} h\right) L_{j}\left(c_{i} c_{k}\right) b_{k}\left(\hat{U}_{D C}^{n}\right)_{j} \\
& +h \sum_{l=0}^{n-1} \sum_{j=1}^{m} K\left(t_{n, i}, t_{l, j}\right) b_{j}\left(\hat{U}_{D C}^{l}\right)_{j} .
\end{aligned}
$$

Here, we have used the fact that

$$
L_{j}\left(c_{k}\right)=\delta_{j, k}= \begin{cases}1, & \text { if } k=j, \\ 0, & \text { otherwise }\end{cases}
$$

Denote

$$
\begin{aligned}
\hat{\mathbf{U}}_{D C}^{n} & :=\left(\left(\hat{U}_{D C}^{n}\right)_{1}, \ldots,\left(\hat{U}_{D C}^{n}\right)_{m}\right)^{T}, \\
\hat{\mathbf{B}}_{D C}^{n} & :=\left(\begin{array}{c}
c_{i} \sum_{k=1}^{m} K\left(t_{n, i}, t_{n}+c_{i} c_{k} h\right) L_{j}\left(c_{i} c_{k}\right) b_{k} \\
(i, j=1, \ldots, m)
\end{array}\right), \\
\hat{\mathbf{B}}_{D C}^{(n, l)} & :=\left(\begin{array}{c}
K\left(t_{n, i}, t_{l, j}\right) b_{j} \\
(i, j=1, \ldots, m)
\end{array}\right) \quad(0 \leq l<n \leq N-1) .
\end{aligned}
$$


Then (2.6) can be written as the following concise form:

$$
\left(\mathbf{I}_{m}-h \hat{\mathbf{B}}_{D C}^{n}\right) \hat{\mathbf{U}}_{D C}^{n}=\mathbf{G}_{D C}^{n}+h \sum_{l=0}^{n-1} \hat{\mathbf{B}}_{D C}^{(n, l)} \hat{\mathbf{U}}_{D C}^{l} .
$$

Similarly to Section 2.1.1, if $g \in C(I)$ and $K \in C(D)$, then there exists a constant $\hat{h}_{D C}>0$ so that for any $h \in\left(0, \hat{h}_{D C}\right)$, the linear algebraic system (2.8) has a unique solution $\hat{\mathbf{U}}_{D C}^{n}$ for all $n=0, \ldots, N-1$. Hence there exists a unique FDC solution $\hat{u}_{D C} \in S_{m-1}^{(-1)}\left(I_{h}\right)$ for (1.1) whose local representation on $\sigma_{n}$ is given by (2.5).

\subsection{DG approximations}

The DG method was considered in $[5,20]$ to solve (1.1). However, these two papers mainly focus on high accuracy methods, including Richardson extrapolation methods, interpolation correction methods and iterative correction methods, and the convergence analysis is based on the operator theory. In this section, we will introduce the DG scheme for (1.1) again, and give the convergence analysis anew without operator theory.

\subsubsection{DG schemes}

For the DG method, we are looking for an approximate solution $u_{D G} \in S_{m-1}^{(-1)}\left(I_{h}\right)$ for (1.1), such that for $\forall \phi \in S_{m-1}^{(-1)}\left(I_{h}\right)$,

$$
\begin{aligned}
& \int_{t_{n}}^{t_{n+1}} u_{D G}(s) \phi(s) d s \\
= & \int_{t_{n}}^{t_{n+1}} g(s) \phi(s) d s+\int_{t_{n}}^{t_{n+1}}\left(\int_{0}^{s} K(s, v) u_{D G}(v) d v\right) \phi(s) d s,
\end{aligned}
$$

i.e.,

$$
\begin{aligned}
& h \int_{0}^{1} u_{D G}\left(t_{n}+s h\right) \phi\left(t_{n}+s h\right) d s \\
= & h \int_{0}^{1} g\left(t_{n}+s h\right) \phi\left(t_{n}+s h\right) d s \\
& +h \int_{0}^{1}\left(\int_{0}^{t_{n}+s h} K\left(t_{n}+s h, v\right) u_{D G}(v) d v\right) \phi\left(t_{n}+s h\right) d s \\
= & h \int_{0}^{1} g\left(t_{n}+s h\right) \phi\left(t_{n}+s h\right) d s \\
& +h^{2} \int_{0}^{1}\left(\int_{0}^{s} K\left(t_{n}+s h, t_{n}+v h\right) u_{D G}\left(t_{n}+v h\right) d v\right) \phi\left(t_{n}+s h\right) d s \\
& +h^{2} \sum_{l=0}^{n-1} \int_{0}^{1}\left(\int_{0}^{1} K\left(t_{n}+s h, t_{l}+v h\right) u_{D G}\left(t_{l}+v h\right) d v\right) \phi\left(t_{n}+s h\right) d s .
\end{aligned}
$$


It is obvious that the exact solution $u$ also satisfies (2.9), i.e.,

$$
\begin{aligned}
& \int_{t_{n}}^{t_{n+1}} u(s) \eta(s) d s \\
= & \int_{t_{n}}^{t_{n+1}} g(s) \eta(s) d s+\int_{t_{n}}^{t_{n+1}} \int_{0}^{s} K(s, v) u(v) d v \eta(s) d s, \quad \forall \eta \in \mathcal{P}_{m-1} .
\end{aligned}
$$

Let

$$
e_{D G}(t):=u(t)-u_{D G}(t) .
$$

Then by (2.11) and (2.9), for $0 \leq n \leq N-1$ and $\forall \eta \in \mathcal{P}_{m-1}$,

$$
\begin{aligned}
& B_{n}\left(e_{D G}, \eta\right) \\
:= & \int_{t_{n}}^{t_{n+1}} e_{D G}(s) \eta(s) d s-\int_{t_{n}}^{t_{n+1}} \int_{0}^{s} K(s, v) e_{D G}(v) d v \eta(s) d s=0,
\end{aligned}
$$

which means that the DG error $e_{D G}(t)$ has the orthogonality property in each subinterval $\sigma_{n}$.

Since $u_{D G} \in S_{m-1}^{(-1)}\left(I_{h}\right)$, the local representation of the DG solution on the subinterval $\sigma_{n}$ can be written as

$$
u_{D G}\left(t_{n}+s h\right)=\sum_{j=0}^{m-1} P_{j}(s)\left(U_{D G}^{n}\right)_{j}, \quad s \in(0,1],
$$

where $P_{j}(s)(j=0, \ldots, m-1)$ denote the 'shifted' Legendre polynomials of degree $j$ on $[0,1]$, and $\left(U_{D G}^{n}\right)_{j}$ are unknowns to be determined. Thus, by (2.10) and (2.13), for $i=0, \ldots, m-1$,

$$
\begin{aligned}
& \sum_{j=0}^{m-1} \int_{0}^{1} P_{j}(s) P_{i}(s) d s\left(U_{D G}^{n}\right)_{j}=\int_{0}^{1} g\left(t_{n}+s h\right) P_{i}(s) d s \\
& \quad+h \sum_{j=0}^{m-1}\left[\int_{0}^{1}\left(\int_{0}^{s} K\left(t_{n}+s h, t_{n}+v h\right) P_{j}(v) d v\right) P_{i}(s) d s\right]\left(U_{D G}^{n}\right)_{j} \\
& \quad+h \sum_{l=0}^{n-1} \sum_{j=0}^{m-1}\left[\int_{0}^{1}\left(\int_{0}^{1} K\left(t_{n}+s h, t_{l}+v h\right) P_{j}(v) d v\right) P_{i}(s) d s\right]\left(U_{D G}^{l}\right)_{j} .
\end{aligned}
$$

Denote

$$
\begin{aligned}
& \mathbf{G}_{D G}^{n}:=\left(\int_{0}^{1} g\left(t_{n}+s h_{n}\right) P_{0}(s) d s, \ldots, \int_{0}^{1} g\left(t_{n}+s h_{n}\right) P_{m-1}(s) d s\right)^{T}, \\
& \mathbf{U}_{D G}^{n}:=\left(\left(U_{D G}^{n}\right)_{0}, \ldots,\left(U_{D G}^{n}\right)_{m-1}\right)^{T}, \quad \mathbf{A}_{D G}^{n}:=\left(\begin{array}{c}
\int_{0}^{1} P_{j}(s) P_{i}(s) d s \\
(i, j=0, \ldots, m-1)
\end{array}\right),
\end{aligned}
$$




$$
\begin{aligned}
\mathbf{B}_{D G}^{n} & :=\left(\int_{0}^{1}\left(\int_{0}^{s} K\left(t_{n}+s h, t_{n}+v h\right) P_{j}(v) d v\right) P_{i}(s) d s\right), \\
\mathbf{B}_{D G}^{(n, l)} & :=\left(\int_{0}^{1}\left(\begin{array}{c}
1 \\
\left.\int_{0} K\left(t_{n}+s h, t_{l}+v h\right) P_{j}(v) d v\right) P_{i}(s) d s \\
(i, j=0, \ldots, m-1)
\end{array}\right) \quad(0 \leq l<n \leq N-1) .\right.
\end{aligned}
$$

Then (2.14) can be written as

$$
\left(\mathbf{A}_{D G}^{n}-h \mathbf{B}_{D G}^{n}\right) \mathbf{U}_{D G}^{n}=\mathbf{G}_{D G}^{n}+h \sum_{l=0}^{n-1} \mathbf{B}_{D G}^{(n, l)} \mathbf{U}_{D G}^{l} .
$$

By [3, Proposition A.1], the matrix

$$
\mathbf{A}_{D G}^{n}:=\left(\begin{array}{cccc}
\int_{0}^{1} P_{i}(s) P_{j}(s) d s \\
(i, j=0, \ldots, m-1)
\end{array}\right)=\left(\begin{array}{cccc}
1 & & & \\
& \frac{1}{3} & & \\
& & \ddots & \\
& & & \frac{1}{2 m-1}
\end{array}\right)
$$

is nonsingular. Therefore, similarly to Section 2.1.1, if $g \in C(I)$ and $K \in C(D)$, there exists a constant $h_{D G}>0$ so that for any $h \in\left(0, h_{D G}\right)$, the linear algebraic system (2.15) has a unique solution $\mathbf{U}_{D G}^{n}$ for all $n=0, \ldots, N-1$. Hence (2.9) determines a unique DG solution $u_{D G} \in S_{m-1}^{(-1)}\left(I_{h}\right)$ for (1.1) whose local representation on $\sigma_{n}$ is given by (2.13).

Remark 2.1. Note that if a different set of basis functions is employed, say $\left\{L_{j}(s)\right\}_{j=1}^{m}$, then there exists a nonsingular matrix $\mathbf{Q}_{D G}$, such that

$$
\left(L_{1}(s), \ldots, L_{m}(s)\right)^{T}=\left(\mathbf{Q}_{D G}\right)^{T}\left(P_{0}(s), \ldots, P_{m-1}(s)\right)^{T} .
$$

Denote the corresponding solution as $\tilde{u}_{D G}$, then (2.13) becomes

$$
\tilde{u}_{D G}\left(t_{n}+s h\right)=\sum_{j=1}^{m} L_{j}(s)\left(\tilde{U}_{D G}^{n}\right)_{j},
$$

where $\left(\tilde{U}_{D G}^{n}\right)_{j}$ are unknowns to be determined. Denote

$$
\begin{aligned}
& \tilde{\mathbf{G}}_{D G}^{n}:=\left(\int_{0}^{1} g\left(t_{n}+s h\right) L_{1}(s) d s, \ldots, \int_{0}^{1} g\left(t_{n}+s h\right) L_{m}(s) d s\right)^{T}, \\
& \tilde{\mathbf{U}}_{D G}^{n}:=\left(\left(\tilde{U}_{D G}^{n}\right)_{1}, \ldots,\left(\tilde{U}_{D G}^{n}\right)_{m}\right)^{T}, \quad \tilde{\mathbf{A}}_{D G}^{n}:=\left(\begin{array}{c}
\int_{0}^{1} L_{j}(s) L_{i}(s) d s \\
(i, j=1, \ldots, m)
\end{array}\right),
\end{aligned}
$$




$$
\begin{aligned}
& \tilde{\mathbf{B}}_{D G}^{n}:=\left(\begin{array}{c}
\int_{0}^{1}\left(\int_{0}^{s} K\left(t_{n}+s h, t_{n}+v h\right) L_{j}(v) d v\right) L_{i}(s) d s \\
(i, j=1, \ldots, m)
\end{array}\right), \\
& \tilde{\mathbf{B}}_{D G}^{(n, l)}:=\left(\begin{array}{c}
\int_{0}^{1}\left(\int_{0}^{1} K\left(t_{n}+s h, t_{l}+v h\right) L_{j}(v) d v\right) L_{i}(s) d s \\
(i, j=1, \ldots, m)
\end{array}\right) \quad(0 \leq l<n \leq N-1) .
\end{aligned}
$$

Then (2.15) becomes

$$
\left(\tilde{\mathbf{A}}_{D G}^{n}-h \tilde{\mathbf{B}}_{D G}^{n}\right) \tilde{\mathbf{U}}_{D G}^{n}=\tilde{\mathbf{G}}_{D G}^{n}+h \sum_{l=0}^{n-1} \tilde{\mathbf{B}}_{D G}^{(n, l)} \tilde{\mathbf{U}}_{D G}^{l},
$$

which can be rewritten as

$$
\left(\mathbf{A}_{D G}^{n}-h \mathbf{B}_{D G}^{n}\right) \mathbf{Q}_{D G} \tilde{\mathbf{U}}_{D G}^{n}=\mathbf{G}_{D G}^{n}+h \sum_{l=0}^{n-1} \mathbf{B}_{D G}^{(n, l)} \mathbf{Q}_{D G} \tilde{\mathbf{U}}_{D G}^{l}
$$

By the uniqueness of the solution, we obtain that $\mathbf{U}_{D G}^{n}=\mathbf{Q}_{D G} \tilde{\mathbf{U}}_{D G}^{n}$, so on the subinterval $\sigma_{n}$, the DG solution based on the basis functions $L_{j}(s)$ is

$$
\begin{aligned}
\tilde{u}_{D G}\left(t_{n}+s h\right) & =\sum_{j=1}^{m} L_{j}(s)\left(\tilde{U}_{D G}^{n}\right)_{j}=\left(L_{1}(s), \ldots, L_{m}(s)\right)\left(\begin{array}{c}
\left(\tilde{U}_{D G}^{n}\right)_{1} \\
\vdots \\
\left(\tilde{U}_{D G}^{n}\right)_{m}
\end{array}\right) \\
& =\left(P_{0}(s), \ldots, P_{m-1}(s)\right) \mathbf{Q}_{D G}\left(\mathbf{Q}_{D G}\right)^{-1}\left(\begin{array}{c}
\left(U_{D G}^{n}\right)_{0} \\
\vdots \\
\left(U_{D G}^{n}\right)_{m-1}
\end{array}\right) \\
& =\left(P_{0}(s), \ldots, P_{m-1}(s)\right)\left(\begin{array}{c}
\left(U_{D G}^{n}\right)_{0} \\
\vdots \\
\left(U_{D G}^{n}\right)_{m-1} \\
\end{array}\right) \\
& =\sum_{j=0}^{m-1} P_{j}(s)\left(U_{D G}^{n}\right)_{j}=u_{D G}\left(t_{n}+s h\right),
\end{aligned}
$$

which means that whatever the choice of basis functions, the resulting DG solutions are equivalent.

\subsubsection{QDG schemes and the relationship with DC schemes}

The QDG scheme is obtained from the DG scheme (2.14) by approximating the inner product by suitable numerical quadrature formulas (see [4]). On $\sigma_{n}$, suppose that the quadrature nodes and weights are based on $\left\{d_{i}\right\}_{i=1}^{q}$ and $\left\{w_{i}\right\}_{i=1}^{q}$, respectively, where 
$q \geq m, 0 \leq d_{1}<\cdots<d_{q} \leq 1$, and at least $m$ weights are nonzero. Then comparing with (2.14), but now employing the basis functions $L_{j}(s)$, we have

$$
\begin{aligned}
& \sum_{j=1}^{m} \sum_{k=1}^{q} L_{j}\left(d_{k}\right) L_{i}\left(d_{k}\right) w_{k}\left(\bar{U}_{D G}^{n}\right)_{j}=\sum_{k=1}^{q} g\left(t_{n}+d_{k} h\right) L_{i}\left(d_{k}\right) w_{k} \\
& \quad+h \sum_{k=1}^{q}\left(\sum_{j=1}^{m} \int_{0}^{d_{k}} K\left(t_{n}+d_{k} h, t_{n}+v h\right) L_{j}(v) d v\left(\bar{U}_{D G}^{n}\right)_{j}\right) L_{i}\left(d_{k}\right) w_{k} \\
& \quad+h \sum_{k=1}^{q} \sum_{l=0}^{n-1}\left(\sum_{j=1}^{m} \int_{0}^{1} K\left(t_{n}+d_{k} h, t_{l}+v h\right) L_{j}(v) d v\left(\bar{U}_{D G}^{l}\right)_{j}\right) L_{i}\left(d_{k}\right) w_{k} .
\end{aligned}
$$

Now take the special case with $q=m$ and $d_{k}=c_{k}$. Then

$$
\begin{aligned}
& \sum_{j=1}^{m} \sum_{k=1}^{m} L_{j}\left(c_{k}\right) L_{i}\left(c_{k}\right) w_{k}\left(\bar{U}_{D G}^{n}\right)_{j}=\sum_{k=1}^{m} g\left(t_{n}+c_{k} h\right) L_{i}\left(c_{k}\right) w_{k} \\
& \quad+h \sum_{k=1}^{m}\left(\sum_{j=1}^{m} \int_{0}^{c_{k}} K\left(t_{n}+c_{k} h, t_{n}+v h\right) L_{j}(v) d v\left(\bar{U}_{D G}^{n}\right)_{j}\right) L_{i}\left(c_{k}\right) w_{k} \\
& \quad+h \sum_{k=1}^{m} \sum_{l=0}^{n-1}\left(\sum_{j=1}^{m} \int_{0}^{1} K\left(t_{n}+c_{k} h, t_{l}+v h\right) L_{j}(v) d v\left(\bar{U}_{D G}^{l}\right)_{j}\right) L_{i}\left(c_{k}\right) w_{k} .
\end{aligned}
$$

By (2.7), for $w_{i} \neq 0$, we obtain

$$
\begin{aligned}
\left(\bar{U}_{D G}^{n}\right)_{i}= & g\left(t_{n, i}\right)+h \sum_{j=1}^{m} \int_{0}^{c_{i}} K\left(t_{n, i}, t_{n}+v h\right) L_{j}(v) d v\left(\bar{U}_{D G}^{n}\right)_{j} \\
& +h \sum_{l=0}^{n-1} \sum_{j=1}^{m} \int_{0}^{1} K\left(t_{n, i}, t_{l}+v h\right) L_{j}(v) d v\left(\bar{U}_{D G}^{l}\right)_{j},
\end{aligned}
$$

which is exactly the DC scheme (2.3), so we have proved the following theorem.

Theorem 2.1. Suppose that the inner products in (2.14) are approximated by $m$-point quadrature formulas with nonzero weights $w_{1}, \ldots, w_{m}$ and nodes $0<d_{1}<\cdots<d_{m} \leq 1$. Then the resulting QDG scheme is identical to the DC scheme of Section 2.1.1 with the collocation parameters $\left\{c_{i}\right\}_{i=1}^{m}=\left\{d_{i}\right\}_{i=1}^{m}$ whatever the choice of weights.

\subsubsection{FDG schemes and the relationship with FDC schemes}

The FDG scheme is obtained from the QDG scheme (2.17) by approximating the integral by suitable numerical quadrature formulas. On $\sigma_{n}$, we choose interpolatory 
$m$-point quadrature formulas whose abscissas are based on the $m$ collocation parameters $\left\{c_{i}\right\}$, and denote $b_{i}$ as the corresponding weights. Then comparing with (2.17), we have

$$
\begin{aligned}
\left(\hat{U}_{D G}^{n}\right)_{i}= & g\left(t_{n, i}\right)+h c_{i} \sum_{j=1}^{m} \sum_{k=1}^{m} K\left(t_{n, i}, t_{n}+c_{i} c_{k} h\right) L_{j}\left(c_{i} c_{k}\right) b_{k}\left(\hat{U}_{D G}^{n}\right)_{j} \\
& +h \sum_{l=0}^{n-1} \sum_{j=1}^{m} K\left(t_{n, i}, t_{l, j}\right) b_{j}\left(\hat{U}_{D G}^{l}\right)_{j},
\end{aligned}
$$

which is exactly the FDC scheme (2.6), so we have proved the following theorem.

Theorem 2.2. Assume that the conditions of Theorem 2.1 hold. Then the resulting FDG scheme is identical to the FDC scheme in Section 2.1.2.

\subsection{Error analysis for DC approximations}

Since in Sections 2.2.2 and 2.2.3, we have shown that for the special choice of the numerical quadrature formula, the resulting QDG and FDG schemes are identical to the DC and FDC schemes, respectively, so in this subsection, we will review the convergence results of the DC (which is also QDG) and the FDC (which is also FDG) methods in [1, Chapter 2], but not give the proofs.

\subsubsection{Error analysis for DC methods}

Theorem 2.3 ([1, Theorem 2.2.3]). Assume:

(a) The given functions describing the VIE (1.1) satisfy $g \in C^{m}(I)$ and $K \in C^{m}(D)$.

(b) $u$ and $u_{D C} \in S_{m-1}^{(-1)}\left(I_{h}\right)$ are the exact solution of (1.1) and the DC solution defined by the collocation equation (2.1) with $h<h_{D C}$.

Then for any set $X_{h}$ of collocation points with $0 \leq c_{1}<\cdots<c_{m} \leq 1$,

$$
\left\|u-u_{D C}\right\|_{\infty}:=\sup _{t \in I}\left|u(t)-u_{D C}(t)\right| \leq C_{D G} M_{m} h^{m},
$$

where $M_{m}:=\max _{t \in I}\left|u^{(m)}(t)\right|$, and the constant $C_{D C}$ depends on $\left\{c_{i}\right\}$, but not on $h$ and $N$.

\subsubsection{Error analysis for FDC methods}

Theorem 2.4 ([1, Theorem 2.2.11]). Under the conditions of Theorem 2.3, assume that the collocation equation defining the exact collocation solution $u_{D C} \in S_{m-1}^{(-1)}\left(I_{h}\right)$ is discretised by using interpolatory m-point quadrature formulas whose abscissas are based on the collocation parameters $\left\{c_{i}\right\}$ and $h<\hat{h}_{D C}$. Then the resulting FDC solution $\hat{u}_{D C}$ has the same convergence property as $u_{D C}$. 


\subsection{Error analysis for DG methods}

Theorem 2.5. Assume:

(a) The given functions describing the VIE (1.1) satisfy $g \in C^{m}(I)$ and $K \in C^{m}(D)$.

(b) $u$ and $u_{D G} \in S_{m-1}^{(-1)}\left(I_{h}\right)$ are the exact solution of (1.1) and the DG solution defined by (2.9) with $h<h_{D G}$.

Then

$$
\left\|u-u_{D G}\right\|_{\infty}:=\sup _{t \in I}\left|u(t)-u_{D G}(t)\right| \leq C_{D G} M_{m} h^{m},
$$

where $C_{D G}$ is a constant independent of $h$ and $N$.

Proof. Assumption (a) implies $u \in C^{m}(I)$ (see [1, Theorem 2.1.3]), so by [3, Lemma 2.3], we have

$$
u\left(t_{n}+s h\right)=\sum_{j=0}^{m-1} P_{j}(s) \hat{u}_{j}^{n}+h^{m} R_{m, n}(s), \quad s \in(0,1],
$$

where

$$
\hat{u}_{j}^{n}=\int_{0}^{1} P_{j}(s) u\left(t_{n}+s h\right) d s\left(\int_{0}^{1}\left(P_{j}(s)\right)^{2} d s\right)^{-1}
$$

is the best $L^{2}$ degree $m-1$ polynomial approximation of $u\left(t_{n}+s h\right)$. The remainder term is bounded by

$$
\left|R_{m, n}(s)\right| \leq \frac{\left|u^{(m)}\left(\zeta_{n}\right)\right|}{m !} \text { for some } \quad \zeta_{n} \in\left(t_{n}, t_{n+1}\right) .
$$

Therefore, by (2.13) and (2.19), we obtain

$$
e_{D G}\left(t_{n}+s h\right)=\sum_{j=0}^{m-1} P_{j}(s)\left(\varepsilon_{D G}^{n}\right)_{j}+h^{m} R_{m, n}(s),
$$

where $\left(\varepsilon_{D G}^{n}\right)_{j}:=\hat{u}_{j}^{n}-\left(U_{D G}^{n}\right)_{j}$. By (2.12) and (2.20), for $i=0, \ldots, m-1$,

$$
\begin{aligned}
& \int_{0}^{1}\left(\sum_{j=0}^{m-1} P_{j}(s)\left(\varepsilon_{D G}^{n}\right)_{j}+h^{m} R_{m, n}(s)\right) P_{i}(s) d s \\
= & h \int_{0}^{1}\left[\int_{0}^{s} K\left(t_{n}+s h, t_{n}+v h\right)\left(\sum_{j=0}^{m-1} P_{j}(v)\left(\varepsilon_{D G}^{n}\right)_{j}+h^{m} R_{m, n}(v)\right) d v\right] P_{i}(s) d s \\
& +h \sum_{l=0}^{n-1} \int_{0}^{1}\left[\int_{0}^{1} K\left(t_{n}+s h, t_{l}+v h\right)\left(\sum_{j=0}^{m-1} P_{j}(v)\left(\varepsilon_{D G}^{l}\right)_{j}+h^{m} R_{m, l}(v)\right) d v\right] P_{i}(s) d s,
\end{aligned}
$$



i.e.,

$$
\begin{aligned}
& \sum_{j=0}^{m-1}\left(\int_{0}^{1} P_{j}(s) P_{i}(s) d s\right)\left(\varepsilon_{D G}^{n}\right)_{j} \\
= & h \sum_{j=0}^{m-1}\left[\int_{0}^{1}\left(\int_{0}^{s} K\left(t_{n}+s h, t_{n}+v h\right) P_{j}(v) d v\right) P_{i}(s) d s\right]\left(\varepsilon_{D G}^{n}\right)_{j} \\
& +h \sum_{l=0}^{n-1} \sum_{j=0}^{m-1}\left[\int_{0}^{1}\left(\int_{0}^{1} K\left(t_{n}+s h, t_{l}+v h\right) P_{j}(v) d v\right) P_{i}(s) d s\right]\left(\varepsilon_{D G}^{l}\right)_{j} \\
& +h^{m}\left(\rho_{D G}^{n}\right)_{i}+\sum_{l=0}^{n-1} h^{m+1}\left(\rho_{D G}^{l}\right)_{i},
\end{aligned}
$$

where

$$
\begin{aligned}
\left(\rho_{D G}^{n}\right)_{i}:= & h \int_{0}^{1}\left(\int_{0}^{s} K\left(t_{n}+s h, t_{n}+v h\right) R_{m, n}(v) d v\right) P_{i}(s) d s \\
& -\int_{0}^{1} R_{m, n}(s) P_{i}(s) d s, \\
\left(\rho_{D G}^{(n, l)}\right)_{i}:= & \int_{0}^{1}\left(\int_{0}^{1} K\left(t_{n}+s h, t_{l}+v h\right) R_{m, l}(v) d v\right) P_{i}(s) d s .
\end{aligned}
$$

Denote

$$
\begin{aligned}
\varepsilon_{D G}^{n} & :=\left(\left(\varepsilon_{D G}^{n}\right)_{0}, \ldots,\left(\varepsilon_{D G}^{n}\right)_{m-1}\right)^{T}, \\
\boldsymbol{\rho}_{D G}^{n} & :=\left(\left(\rho_{D G}^{n}\right)_{0}, \ldots,\left(\rho_{D G}^{n}\right)_{m-1}\right)^{T}, \\
\boldsymbol{\rho}_{D G}^{(n, l)} & :=\left(\left(\rho_{D G}^{(n, l)}\right)_{0}, \ldots,\left(\rho_{D G}^{(n, l)}\right)_{m-1}\right)^{T} .
\end{aligned}
$$

Then

$$
\left(\mathbf{A}_{D G}^{n}-h \mathbf{B}_{D G}^{n}\right) \varepsilon_{D G}^{n}=h \sum_{l=0}^{n-1} \mathbf{B}_{D G}^{(n, l)} \varepsilon_{D G}^{l}+h^{m} \boldsymbol{\rho}_{D G}^{n}+h^{m+1} \sum_{l=0}^{n-1} \boldsymbol{\rho}_{D G}^{(n, l)} .
$$

Since $h<h_{D G}$, similarly to Section 2.2.1, we know that there exists a constant $D_{1}$, such that $\left\|\left(\mathbf{A}_{D G}^{n}-h \mathbf{B}_{D G}^{n}\right)^{-1}\right\|_{\infty} \leq D_{1}$, and

$$
\begin{aligned}
\left\|\varepsilon_{D G}^{n}\right\|_{\infty} \leq D_{1}\left[\sum_{l=0}^{n-1} h m \bar{K} \bar{P}^{2}\left\|\varepsilon_{D G}^{l}\right\|_{\infty}+h^{m}\left(h \bar{K} \bar{P} \frac{\left\|u^{(m)}\right\|_{\infty}}{m !}+\bar{P} \frac{\left\|u^{(m)}\right\|_{\infty}}{m !}\right)\right. \\
\left.\quad+h^{m+1} \sum_{l=0}^{n-1} \bar{K} \bar{P} \frac{\left\|u^{(m)}\right\|_{\infty}}{m !}\right] \\
\leq D_{1}\left[\sum_{l=0}^{n-1} h m \bar{K} \bar{P}^{2}\left\|\varepsilon_{D G}^{l}\right\|_{\infty}+\bar{P}(2 T \bar{K}+1) \frac{M_{m}}{m !} h^{m}\right],
\end{aligned}
$$


where

$$
\bar{K}:=\max _{0 \leq s \leq t \leq T}|K(t, s)|, \quad \bar{P}:=\max _{s \in[0,1], 0 \leq j \leq m-1}\left|P_{j}(s)\right| .
$$

The discrete Gronwall inequality (see [1, Corollay 2.1.19]) yields

$$
\left\|\varepsilon_{D G}^{n}\right\|_{\infty} \leq \frac{D_{1} \bar{P}(2 T \bar{K}+1)}{m !} \exp \left(D_{1} \bar{K} \bar{P}^{2} m T\right) M_{m} h^{m},
$$

which together with (2.20) yield

$$
\left|e_{D G}\left(t_{n}+s h\right)\right| \leq \frac{D_{1} m \bar{P}^{2}(2 T \bar{K}+1) \exp \left(D_{1} \bar{K} \bar{P}^{2} m T\right)+1}{m !} M_{m} h^{m} .
$$

This completes the proof.

Remark 2.2. By Theorems 2.3 and 2.5, we see that the convergence order of the DG method is as same as the order of the DC method.

\section{Continuous approximations}

\subsection{CC approximations}

In [13], CC methods are investigated thoroughly for (1.1). In order to establish the relationship between CC and CG methods, in this subsection, we will review CC methods first.

\subsubsection{CC schemes}

At $t=t_{n, i}$, the collocation equation reads as

$$
u_{C C}(t)=g(t)+\int_{0}^{t} K(t, s) u_{C C}(s) d s, \quad t \in X_{h}
$$

with $u_{C C}(0)=g(0)$.

Since $u_{C C} \in S_{m}^{(0)}\left(I_{h}\right)$, the local representation of the CC solution $u_{C C}$ on the subinterval $\sigma_{n}$ can be written as

$$
u_{C C}\left(t_{n}+s h\right)=\sum_{j=0}^{m} l_{j}(s)\left(U_{C C}^{n}\right)_{j}, \quad s \in[0,1],
$$

where

$$
\left(U_{C C}^{n}\right)_{0}:=u_{C C}\left(t_{n}\right), \quad\left(U_{C C}^{n}\right)_{j}:=u_{C C}\left(t_{n, j}\right) \quad \text { for } \quad j=1, \ldots, m
$$

and

$$
l_{0}(s):=\prod_{i=1}^{m} \frac{s-c_{i}}{-c_{i}}, \quad l_{j}(s):=\frac{s}{c_{j}} \prod_{i=1, i \neq j}^{m} \frac{s-c_{i}}{c_{j}-c_{i}} .
$$


Therefore, by (3.1) and (3.2),

$$
\begin{aligned}
\left(U_{C C}^{n}\right)_{i}= & g\left(t_{n, i}\right)+\int_{0}^{t_{n, i}} K\left(t_{n, i}, s\right) u_{C C}(s) d s \\
= & g\left(t_{n, i}\right)+h \int_{0}^{c_{i}} K\left(t_{n, i}, t_{n}+s h\right) u_{C C}\left(t_{n}+s h\right) d s \\
& +h \sum_{l=0}^{n-1} \int_{0}^{1} K\left(t_{n, i}, t_{l}+s h\right) u_{C C}\left(t_{l}+s h\right) d s \\
= & g\left(t_{n, i}\right)+h \int_{0}^{c_{i}} K\left(t_{n, i}, t_{n}+s h\right)\left(\sum_{j=0}^{m} l_{j}(s)\left(U_{C C}^{n}\right)_{j}\right) d s \\
& +h \sum_{l=0}^{n-1} \int_{0}^{1} K\left(t_{n, i}, t_{l}+s h\right)\left(\sum_{j=0}^{m} l_{j}(s)\left(U_{C C}^{l}\right)_{j}\right) d s \\
= & g\left(t_{n, i}\right)+h \int_{0}^{c_{i}} K\left(t_{n, i}, t_{n}+s h\right) l_{0}(s) d s u_{C C}\left(t_{n}\right) \\
& +h \sum_{j=1}^{m} \int_{0}^{c_{i}} K\left(t_{n, i}, t_{n}+s h\right) l_{j}(s) d s\left(U_{C C}^{n}\right)_{j} \\
& +h \sum_{l=0}^{n-1} \int_{0}^{1} K\left(t_{n, i}, t_{l}+s h\right) l_{0}(s) d s u_{C C}\left(t_{l}\right)^{n} \\
& +h \sum_{l=0}^{n-1} \sum_{j=1}^{m} \int_{0}^{1} K\left(t_{n, i}, t_{l}+s h\right) l_{j}(s) d s\left(U_{C C}^{l}\right)_{j} .
\end{aligned}
$$

\section{Denote}

$$
\begin{aligned}
& \mathbf{U}_{C C}^{n}:=\left(\left(U_{C C}^{n}\right)_{1}, \ldots,\left(U_{C C}^{n}\right)_{m}\right)^{T}, \quad \mathbf{e}:=(\underbrace{1, \ldots, 1}_{m})^{T}, \\
& \mathbf{M}_{C C}^{n}:=\operatorname{diag}\left(\begin{array}{c}
\int_{0}^{c_{i}} K\left(t_{n, i}, t_{n}+s h\right) l_{0}(s) d s \\
(i=1, \ldots, m)
\end{array}\right), \\
& \mathbf{B}_{C C}^{n}:=\left(\begin{array}{c}
\int_{0}^{c_{i}} K\left(t_{n, i}, t_{n}+s h\right) l_{j}(s) d s \\
(i, j=1, \ldots, m)
\end{array}\right), \\
& \mathbf{M}_{C C}^{(n, l)}:=\operatorname{diag}\left(\begin{array}{c}
\int_{0}^{1} K\left(t_{n, i}, t_{l}+s h\right) l_{0}(s) d s \\
(i=1, \ldots, m)
\end{array}\right), \\
& \mathbf{B}_{C C}^{(n, l)}:=\left(\begin{array}{c}
\int_{0}^{1} K\left(t_{n, i}, t_{l}+s h\right) l_{j}(s) d s \\
(i, j=1, \ldots, m)
\end{array}\right) \quad(0 \leq l<n \leq N-1) .
\end{aligned}
$$


Then (3.4) can be written as the following concise form:

$$
\begin{aligned}
& \left(\mathbf{I}_{m}-h \mathbf{B}_{C C}^{n}\right) \mathbf{U}_{C C}^{n} \\
= & \mathbf{G}_{D C}^{n}+h \mathbf{M}_{C C}^{n} \mathbf{e} u_{C C}\left(t_{n}\right)+h \sum_{l=0}^{n-1}\left[\mathbf{M}_{C C}^{(n, l)} \mathbf{e} u_{C C}\left(t_{l}\right)+\mathbf{B}_{C C}^{(n, l)} \mathbf{U}_{C C}^{l}\right] .
\end{aligned}
$$

Similarly to Section 2.1.1, if $g \in C(I)$ and $K \in C(D)$, there exists a constant $h_{C C}>0$ so that for any $h \in\left(0, h_{C C}\right)$, the linear algebraic system (3.5) has a unique solution $\mathbf{U}_{C C}^{n}$ for all $n=0, \ldots, N-1$. Hence (3.1) determines a unique CC solution $u_{C C} \in S_{m}^{(0)}\left(I_{h}\right)$ for (1.1) whose local representation on $\left[t_{n}, t_{n+1}\right]$ is given by (3.2).

\subsubsection{FCC schemes}

Similarly to Section 2.1.2, denote $\hat{u}_{C C} \in S_{m}^{(0)}\left(I_{h}\right)$ as the FCC solution, then the local representation of $\hat{u}_{C C}$ on the subinterval $\sigma_{n}$ can be written as

$$
\hat{u}_{C C}\left(t_{n}+s h\right)=\sum_{j=0}^{m} l_{j}(s)\left(\hat{U}_{C C}^{n}\right)_{j}, \quad s \in[0,1],
$$

where

$$
\left(\hat{U}_{C C}^{n}\right)_{0}:=\hat{u}_{C C}\left(t_{n}\right), \quad\left(\hat{U}_{C C}^{n}\right)_{j}:=\hat{u}_{C C}\left(t_{n, j}\right) \quad \text { for } j=1, \ldots, m .
$$

On $\sigma_{n}$, we choose interpolatory $m+1$-point quadrature formulas whose abscissas are based on $c_{0}:=0$, and the $m$ collocation parameters $\left\{c_{i}\right\}$, and denote

$$
\tilde{b}_{i}:=\int_{0}^{1} l_{i}(s) d s, \quad i=0, \ldots, m
$$

as the corresponding weights. Then by (3.4), we have

$$
\begin{aligned}
\left(\hat{U}_{C C}^{n}\right)_{i}= & g\left(t_{n, i}\right)+h c_{i} \sum_{j=0}^{m} \sum_{k=0}^{m} K\left(t_{n, i}, t_{n}+c_{i} c_{k} h\right) l_{j}\left(c_{i} c_{k}\right) \tilde{b}_{k}\left(\hat{U}_{C C}^{n}\right)_{j} \\
& +h \sum_{l=0}^{n-1} \sum_{j=0}^{m} \sum_{k=0}^{m} K\left(t_{n, i}, t_{l}+c_{k} h\right) l_{j}\left(c_{k}\right) \tilde{b}_{k}\left(\hat{U}_{C C}^{l}\right)_{j} \\
= & g\left(t_{n, i}\right)+h c_{i} \sum_{j=0}^{m} \sum_{k=0}^{m} K\left(t_{n, i}, t_{n}+c_{i} c_{k} h\right) l_{j}\left(c_{i} c_{k}\right) \tilde{b}_{k}\left(\hat{U}_{C C}^{n}\right)_{j} \\
& +h \sum_{l=0}^{n-1} \sum_{j=0}^{m} K\left(t_{n, i}, t_{l, j}\right) \tilde{b}_{j}\left(\hat{U}_{C C}^{l}\right)_{j},
\end{aligned}
$$

where we have used the fact that

$$
l_{j}\left(c_{k}\right)=\delta_{j, k}= \begin{cases}1, & \text { if } k=j \\ 0, & \text { otherwise }\end{cases}
$$


Denote

$$
\begin{aligned}
\hat{\mathbf{U}}_{C C}^{n} & :=\left(\left(\hat{U}_{C C}^{n}\right)_{1}, \ldots,\left(\hat{U}_{C C}^{n}\right)_{m}\right)^{T}, \\
\hat{\mathbf{M}}_{C C}^{n} & :=\operatorname{diag}\left(\begin{array}{c}
c_{i} \sum_{k=0}^{m} K\left(t_{n, i}, t_{n}+c_{i} c_{k} h\right) l_{0}\left(c_{i} c_{k}\right) \tilde{b}_{k} \\
(i=1, \ldots, m)
\end{array}\right), \\
\hat{\mathbf{B}}_{C C}^{n} & :=\left(\begin{array}{c}
c_{i} \sum_{k=0}^{m} K\left(t_{n, i}, t_{n}+c_{i} c_{k} h\right) l_{j}\left(c_{i} c_{k}\right) \tilde{b}_{k} \\
(i, j=1, \ldots, m)
\end{array}\right), \\
\hat{\mathbf{M}}_{C C}^{(n, l)} & :=\operatorname{diag}\left(\begin{array}{c}
K\left(t_{n, i}, t_{l}\right) \tilde{b}_{0} \\
(i=1, \ldots, m)
\end{array}\right), \\
\hat{\mathbf{B}}_{C C}^{(n, l)} & :=\left(\begin{array}{c}
K\left(t_{n, i}, t_{l, j}\right) \tilde{b}_{j} \\
(i, j=1, \ldots, m)
\end{array}\right) \quad(0 \leq l<n \leq N-1) .
\end{aligned}
$$

Then (3.7) can be written in the following concise form

$$
\begin{aligned}
& \left(\mathbf{I}_{m}-h \hat{\mathbf{B}}_{C C}^{n}\right) \hat{\mathbf{U}}_{C C}^{n} \\
= & \mathbf{G}_{D C}^{n}+h \hat{\mathbf{M}}_{C C}^{n} \mathbf{e} \hat{u}_{C C}\left(t_{n}\right)+h \sum_{l=0}^{n-1}\left[\hat{\mathbf{M}}_{C C}^{(n, l)} \mathbf{e} \hat{u}_{C C}\left(t_{l}\right)+\hat{\mathbf{B}}_{C C}^{(n, l)} \hat{\mathbf{U}}_{C C}^{l}\right] .
\end{aligned}
$$

Similarly to Section 2.1.1, if $g \in C(I)$ and $K \in C(D)$, then there exists a constant $\hat{h}_{C C}>0$ so that for any $h \in\left(0, \hat{h}_{C C}\right)$, the linear algebraic system (3.9) has a unique solution $\hat{\mathbf{U}}_{C C}^{n}$ for all $n=0, \ldots, N-1$. Hence there determines a unique FCC solution $\hat{u}_{C C} \in S_{m}^{(0)}\left(I_{h}\right)$ for (1.1) whose local representation on $\left[t_{n}, t_{n+1}\right]$ is given by (3.6).

\subsection{CG approximations}

\subsubsection{CG schemes}

In the CG method, we are looking for an approximate solution $u_{C G} \in S_{m}^{(0)}\left(I_{h}\right)$ for (1.1) such that for $0 \leq n \leq N-1$ and $\forall \eta \in \mathcal{P}_{m-1}$,

$$
\begin{aligned}
& \int_{t_{n}}^{t_{n+1}} u_{C G}(s) \eta(s) d s \\
= & \int_{t_{n}}^{t_{n+1}} g(s) \eta(s) d s+\int_{t_{n}}^{t_{n+1}} \int_{0}^{s} K(s, v) u_{C G}(v) d v \eta(s) d s .
\end{aligned}
$$

Here, because of the continuity of $u_{C G}(t)$, we have

$$
u_{C G}\left(t_{n-1}\right)=\lim _{t \rightarrow t_{n-1}^{-}} u_{C G}(t)=\lim _{t \rightarrow t_{n-1}^{+}} u_{C G}(t) .
$$


Hence $u_{C G}(t)$ has only $m$ degrees of freedom on each subinterval, so $\eta \in \mathcal{P}_{m-1}$ (see [10]).

It is obvious that the exact solution $u$ also satisfies (3.10), i.e., for $\forall \eta \in \mathcal{P}_{m-1}$,

$$
\begin{aligned}
& \int_{t_{n}}^{t_{n+1}} u(s) \eta(s) d s \\
= & \int_{t_{n}}^{t_{n+1}} g(s) \eta(s) d s+\int_{t_{n}}^{t_{n+1}} \int_{0}^{s} K(s, v) u(v) d v \eta(s) d s .
\end{aligned}
$$

Let

$$
e_{C G}(t):=u(t)-u_{C G}(t) \text {. }
$$

Then by (3.10) and (3.11), for $0 \leq n \leq N-1$ and $\forall \eta \in \mathcal{P}_{m-1}$,

$$
\begin{aligned}
& B_{n}\left(e_{C G}, \eta\right) \\
:= & \int_{t_{n}}^{t_{n+1}} e_{C G}(s) \eta(s) d s-\int_{t_{n}}^{t_{n+1}} \int_{0}^{s} K(s, v) e_{C G}(v) d v \eta(s) d s=0,
\end{aligned}
$$

which means that the CG error $e_{C G}(t)$ has the orthogonality property in each subinterval $\sigma_{n}$.

Since $u_{C G} \in S_{m}^{(0)}\left(I_{h}\right)$, the local representation of the CG solution on the subinterval $\sigma_{n}$ can be written as

$$
u_{C G}\left(t_{n}+s h\right)=\sum_{j=0}^{m} P_{j}(s)\left(U_{C G}^{n}\right)_{j}, \quad s \in[0,1],
$$

where the unknowns $\left(U_{C G}^{n}\right)_{j}$ are to be determined. By (3.10),

$$
\begin{aligned}
& h \int_{0}^{1} u_{C G}\left(t_{n}+s h\right) \eta\left(t_{n}+s h\right) d s \\
= & h \int_{0}^{1} g\left(t_{n}+s h\right) \eta\left(t_{n}+s h\right) d s \\
& +h \int_{0}^{1}\left(\int_{0}^{t_{n}+s h} K\left(t_{n}+s h, v\right) u_{C G}(v) d v\right) \eta\left(t_{n}+s h\right) d s \\
= & h \int_{0}^{1} g\left(t_{n}+s h\right) \eta\left(t_{n}+s h\right) d s \\
& +h^{2} \int_{0}^{1}\left(\int_{0}^{s} K\left(t_{n}+s h, t_{n}+v h\right) u_{C G}\left(t_{n}+v h\right) d v\right) \eta\left(t_{n}+s h\right) d s \\
& +h^{2} \sum_{l=0}^{n-1} \int_{0}^{1}\left(\int_{0}^{1} K\left(t_{n}+s h, t_{l}+v h\right) u_{C G}\left(t_{l}+v h\right) d v\right) \eta\left(t_{n}+s h\right) d s,
\end{aligned}
$$

which together with (3.13) yield that for $i=0, \ldots, m-1$,

$$
\sum_{j=0}^{m}\left(\int_{0}^{1} P_{j}(s) P_{i}(s) d s\right)\left(U_{C G}^{n}\right)_{j}=\int_{0}^{1} g\left(t_{n}+s h\right) P_{i}(s) d s
$$




$$
\begin{aligned}
& +h \sum_{j=0}^{m}\left[\int_{0}^{1}\left(\int_{0}^{s} K\left(t_{n}+s h, t_{n}+v h\right) P_{j}(v) d v\right) P_{i}(s) d s\right]\left(U_{C G}^{n}\right)_{j} \\
& +h \sum_{l=0}^{n-1} \sum_{j=0}^{m}\left[\int_{0}^{1}\left(\int_{0}^{1} K\left(t_{n}+s h, t_{l}+v h\right) P_{j}(v) d v\right) P_{i}(s) d s\right]\left(U_{C G}^{l}\right)_{j} .
\end{aligned}
$$

Denote

$$
\begin{aligned}
& \mathbf{U}_{C G}^{n}:=\left(\left(U_{C G}^{n}\right)_{0}, \ldots,\left(U_{C G}^{n}\right)_{m}\right)^{T}, \\
& \mathbf{A}_{C G}^{n}:=\left(\begin{array}{c}
\int_{0}^{1} P_{j}(s) P_{i}(s) d s \\
(i=0, \ldots, m-1 ; j=0, \ldots, m)
\end{array}\right), \\
& \mathbf{B}_{C G}^{n}:=\left(\begin{array}{c}
\int_{0}^{1}\left(\int_{0}^{s} K\left(t_{n}+s h, t_{n}+v h\right) P_{j}(v) d v\right) P_{i}(s) d s \\
(i=0, \ldots, m-1 ; j=0, \ldots, m)
\end{array}\right), \\
& \mathbf{B}_{C G}^{(n, l)}:=\left(\begin{array}{c}
\int_{0}^{1}\left(\int_{0}^{1} K\left(t_{n}+s h, t_{l}+v h\right) P_{j}(v) d v\right) P_{i}(s) d s \\
(i=0, \ldots, m-1 ; j=0, \ldots, m)
\end{array}\right) \quad(0 \leq l<n \leq N-1) .
\end{aligned}
$$

Note that now the matrices $\mathbf{A}_{C G}^{n}, \mathbf{B}_{C G}^{n}$ and $\mathbf{B}_{C G}^{(n, l)}$ are not square, which are different from the matrices appearing in the DG scheme in Section 2.2.1, where all of the matrices $\mathbf{A}_{D G}^{n}, \mathbf{B}_{D G}^{n}$ and $\mathbf{B}_{D G}^{(n, l)}$ are square. Therefore, here we resort to the continuity, which together with (3.13) and (3.14) yield

$$
\begin{aligned}
& \left(\begin{array}{c}
\left(P_{0}(0), \ldots, P_{m}(0)\right) \\
\left(\mathbf{A}_{C G}^{n}-h \mathbf{B}_{C G}^{n}\right)
\end{array}\right) \mathbf{U}_{C G}^{n} \\
= & \left(\begin{array}{c}
\left(P_{0}(1), \ldots, P_{m}(1)\right) \\
\mathbf{0}_{m \times(m+1)}
\end{array}\right) \mathbf{U}_{C G}^{n-1}+h \sum_{l=0}^{n-1}\left(\begin{array}{c}
\mathbf{0}_{1 \times(m+1)} \\
\mathbf{B}_{C G}^{(n, l)}
\end{array}\right) \mathbf{U}_{C G}^{l}+\left(\begin{array}{c}
0 \\
\mathbf{G}_{D G}^{n}
\end{array}\right) .
\end{aligned}
$$

By the properties of Legendre polynomials and [3, Proposition A.1], the matrix

$$
\overline{\mathbf{A}}_{C G}^{n}:=\left(\begin{array}{c}
\left(P_{0}(0), \ldots, P_{m}(0)\right) \\
\mathbf{A}_{C G}^{n}
\end{array}\right)=\left(\begin{array}{cccc}
1 & -1 & \ldots & (-1)^{m} \\
1 & \ldots & 0 & 0 \\
\vdots & \ddots & \vdots & \vdots \\
0 & \ldots & \frac{1}{2 m-1} & 0
\end{array}\right)
$$

is nonsingular. It can be easily checked that

$$
\left(\overline{\mathbf{A}}_{C G}^{n}\right)^{-1}=\left(\begin{array}{ccccc}
0 & 1 & 0 & \ldots & 0 \\
0 & 0 & 3 & \ldots & 0 \\
\vdots & \vdots & \vdots & \vdots & \vdots \\
0 & 0 & 0 & \ldots & 2 m-1 \\
(-1)^{m} & (-1)^{m-1} & (-1)^{m-2} 3 & \ldots & 2 m-1
\end{array}\right) .
$$


Therefore, similarly to Section 2.1.1, if $g \in C(I)$ and $K \in C(D)$, then there exists a constant $h_{C G}>0$ so that for any $h \in\left(0, h_{C G}\right)$, the linear algebraic system (3.15) has a unique solution $\mathbf{U}_{C G}^{n}$ for all $n=0, \ldots, N-1$. Hence for all sufficiently small $h>0$ (3.10) determines a unique CG solution $u_{C G} \in S_{m}^{(0)}\left(I_{h}\right)$ for (1.1) whose local representation on $\left[t_{n}, t_{n+1}\right]$ is given by (3.13).

Remark 3.1. Similarly to Remark 2.1, it is easy to obtain that whatever the choice of basis functions, the resulting CG solutions are equivalent.

\subsubsection{QCG schemes and the relationship with CC schemes}

Similarly to Section 2.2.2, suppose that the quadrature nodes and weights for the inner products of (3.14) are $\left\{d_{i}\right\}_{i=1}^{q}$ and $\left\{w_{i}\right\}_{i=1}^{q}$, respectively, where $q \geq m, 0 \leq d_{1}<\cdots<$ $d_{q} \leq 1$, and at least $m$ weights are nonzero. Then comparing with (3.14), but now employing the basis functions $l_{j}$ and $L_{j}(s)$, we obtain

$$
\begin{aligned}
& \sum_{j=0}^{m} \sum_{k=1}^{q} l_{j}\left(d_{k}\right) L_{i}\left(d_{k}\right) w_{k}\left(\bar{U}_{C G}^{n}\right)_{j}=\sum_{k=1}^{q} g\left(t_{n}+d_{k} h\right) L_{i}\left(d_{k}\right) w_{k} \\
& \quad+h \sum_{j=0}^{m} \sum_{k=1}^{q}\left(\int_{0}^{d_{k}} K\left(t_{n}+d_{k} h, t_{n}+v h\right) l_{j}(v) d v\right) L_{i}\left(d_{k}\right) w_{k}\left(\bar{U}_{C G}^{n}\right)_{j} \\
& \quad+h \sum_{l=0}^{n-1} \sum_{j=0}^{m} \sum_{k=1}^{q}\left(\int_{0}^{1} K\left(t_{n}+d_{k} h, t_{l}+v h\right) l_{j}(v) d v\right) L_{i}\left(d_{k}\right) w_{k}\left(\bar{U}_{C G}^{l}\right)_{j} .
\end{aligned}
$$

Now take the special case with $q=m$ and $d_{k}=c_{k}$. Then by (3.8), for $i=1, \ldots, m$ and $w_{i} \neq 0$,

$$
\begin{aligned}
\left(\bar{U}_{C G}^{n}\right)_{i}= & g\left(t_{n, i}\right)+h\left(\int_{0}^{c_{i}} K\left(t_{n, i}, t_{n}+v h\right) l_{0}(v) d v\right) \bar{u}_{C G}\left(t_{n}\right) \\
& +h \sum_{j=1}^{m}\left(\int_{0}^{c_{i}} K\left(t_{n, i}, t_{n}+v h\right) l_{j}(v) d v\right)\left(\bar{U}_{C G}^{n}\right)_{j} \\
& +h \sum_{l=0}^{n-1}\left(\int_{0}^{1} K\left(t_{n, i}, t_{l}+v h\right) l_{0}(v) d v\right) \bar{u}_{C G}\left(t_{l}\right) \\
& +h \sum_{l=0}^{n-1} \sum_{j=1}^{m}\left(\int_{0}^{1} K\left(t_{n, i}, t_{l}+v h\right) l_{j}(v) d v\right)\left(\bar{U}_{C G}^{l}\right)_{j},
\end{aligned}
$$

which is exactly the CC scheme (3.4), so we have proved the following theorem.

Theorem 3.1. Suppose that the inner products of (3.14) are approximated by m-point quadrature formulas with nonzero weights $w_{1}, \ldots, w_{m}$ and nodes $0<d_{1}<\cdots<d_{m} \leq 1$. Then the resulting QCG scheme is identical to the CC scheme of Section 3.1.1 with the collocation parameters $\left\{c_{i}\right\}_{i=1}^{m}=\left\{d_{i}\right\}_{i=1}^{m}$ whatever the choice of weights. 


\subsubsection{FCG schemes and the relationship with FCC schemes}

Similarly to Section 3.1.2, comparing with (3.17), we have

$$
\begin{aligned}
\left(\hat{U}_{C G}^{n}\right)_{i}= & g\left(t_{n, i}\right)+h c_{i} \sum_{k=0}^{m} K\left(t_{n, i}, t_{n}+c_{i} c_{k} h\right) l_{0}\left(c_{i} c_{k}\right) \tilde{b}_{k} \hat{u}_{C G}\left(t_{n}\right) \\
& +h c_{i} \sum_{j=1}^{m} \sum_{k=0}^{m} K\left(t_{n, i}, t_{n}+c_{i} c_{k} h\right) l_{j}\left(c_{i} c_{k}\right) \tilde{b}_{k}\left(\hat{U}_{C G}^{n}\right)_{j} \\
& +h \sum_{l=0}^{n-1} K\left(t_{n, i}, t_{l}\right) \tilde{b}_{0} \hat{u}_{C G}\left(t_{l}\right)+h \sum_{l=0}^{n-1} \sum_{j=1}^{m} K\left(t_{n, i}, t_{l, j}\right) \tilde{b}_{j}\left(\hat{U}_{C G}^{l}\right)_{j}
\end{aligned}
$$

which is exactly the FCC scheme (3.7), so we obtain the following theorem.

Theorem 3.2. Assume that the conditions of Theorem 3.1 hold. Then the resulting FCG scheme is identical to the FCC scheme in Section 3.1.2.

\subsection{Error analysis for CC approximations}

Since in Sections 3.2.2 and 3.2.3, we have shown that for the special choice of the numerical quadrature formula, the resulting QCG and FCG methods are identical to the CC and FCC methods, respectively, so in this subsection, we will first review the convergence result of the CC (which is also QCG) method in [13], then give the error analysis for the FCC (which is also FCG) method.

\subsubsection{Error analysis for CC methods}

Theorem 3.3 ([13, Theorem 2.1]). Assume that in (1.1) we have $g \in C^{m+2}(I)$ and $K \in C^{m+2}(D)$. Let $u$ and $u_{C C} \in S_{m}^{(0)}\left(I_{h}\right)$ be the exact solution and the CC solution defined by the collocation equation (3.1) with $h<h_{C C}$. Then the CC solution converges to the exact solution if and only if, the collocation parameters $\left\{c_{i}\right\}$ satisfy the condition

$$
-1 \leq l_{0}(1)=(-1)^{m} \prod_{i=1}^{m} \frac{1-c_{i}}{c_{i}} \leq 1 .
$$

The corresponding attainable global order of convergence is given by

$$
\left\|u-u_{C C}\right\|_{\infty}:=\max _{t \in I}\left|u(t)-u_{C C}(t)\right| \leq C_{C C} \begin{cases}h^{m+1}, & \text { if }-1 \leq \rho_{m}<1, \\ h^{m}, & \text { if } \quad \rho_{m}=1,\end{cases}
$$

where the constant $C_{C C}$ depends on $\left\{c_{i}\right\}$ and the derivatives of the exact solution $u$, but not on $h$ and $N$. 


\subsubsection{Error analysis for FCC methods}

Combining the proofs of the convergence for CC methods (see [13, Theorem 2.1]) and FDC (see [1, Theorem 2.2.11]), we can easily obtain the following theorem.

Theorem 3.4. Under the condition of Theorem 3.3, assume that the CC equation defining the exact collocation solution $u_{C C} \in S_{m}^{(0)}\left(I_{h}\right)$ are discretised by using interpolatory $m+$ 1-point quadrature formulas whose abscissas are based on $c_{0}=0$ and the collocation parameters $\left\{c_{i}\right\}$ and $h<\hat{h}_{C C}$. Then the resulting discretised collocation approximation $\hat{u}_{C C}$ has the same convergence property as $u_{C C}$.

\subsection{Error analysis for CG methods}

Theorem 3.5. Assume that in (1.1) we have $g \in C^{m+2}(I)$ and $K \in C^{m+2}(D)$. Let $u$ and $u_{C G} \in S_{m}^{(0)}\left(I_{h}\right)$ be the exact solution and the CG solution defined by (3.10) with $h<h_{C G}$. Then

$$
\left\|u-u_{C G}\right\|_{\infty}:=\max _{t \in I}\left|u(t)-u_{C G}(t)\right| \leq C_{C G} \begin{cases}h^{m+1}, & \text { if } m \text { is odd, } \\ h^{m}, & \text { if } m \text { is even, }\end{cases}
$$

where the constant $C_{C G}$ depends on the derivatives of the exact solution $u$, but not on $h$ and $N$.

Proof. By the assumption of the regularity on $g$ and $K$, and [3, Lemma 2.3], we have

$$
u\left(t_{n}+s h\right)=\sum_{j=0}^{m} P_{j}(s) \hat{u}_{j}^{n}+h^{m+1} R_{m+1, n}(s),
$$

where $\hat{u}_{j}^{n}$ is the best $L^{2}$ degree $m$ polynomial approximation of $u\left(t_{n}+s h\right)$ defined in the proof of Theorem 2.5. The remainder term is bounded by

$$
\left|R_{m+1, n}(s)\right| \leq \frac{\left|u^{(m+1)}\left(\zeta_{n}^{\prime}\right)\right|}{(m+1) !} \text { for some } \quad \zeta_{n}^{\prime} \in\left(t_{n}, t_{n+1}\right) .
$$

By (3.13) and (3.19),

$$
e_{C G}\left(t_{n}+s h\right)=\sum_{j=0}^{m} P_{j}(s)\left(\varepsilon_{C G}^{n}\right)_{j}+h^{m+1} R_{m+1, n}(s),
$$

where $\left(\varepsilon_{C G}^{n}\right)_{j}:=\hat{u}_{j}^{n}-\left(U_{C G}^{n}\right)_{j}$. By (3.12) and (3.20),

$$
\begin{aligned}
& \int_{0}^{1}\left(\sum_{j=0}^{m} P_{j}(s)\left(\varepsilon_{C G}^{n}\right)_{j}+h^{m+1} R_{m+1, n}(s)\right) P_{i}(s) d s \\
= & \int_{0}^{1}\left[h \int_{0}^{s} K\left(t_{n}+s h, t_{n}+v h\right)\left(\sum_{j=0}^{m} P_{j}(v)\left(\varepsilon_{C G}^{n}\right)_{j}+h^{m+1} R_{m+1, n}(v)\right) d v\right.
\end{aligned}
$$




$$
\left.+h \sum_{l=0}^{n-1} \int_{0}^{1} K\left(t_{n}+s h, t_{l}+v h\right)\left(\sum_{j=0}^{m} P_{j}(v)\left(\varepsilon_{C G}^{l}\right)_{j}+h^{m+1} R_{m+1, l}(v)\right) d v\right] P_{i}(s) d s,
$$

i.e.,

$$
\begin{aligned}
& \sum_{j=0}^{m}\left(\int_{0}^{1} P_{j}(s) P_{i}(s) d s\right)\left(\varepsilon_{C G}^{n}\right)_{j} \\
= & h \sum_{j=0}^{m}\left[\int_{0}^{1}\left(\int_{0}^{s} K\left(t_{n}+s h, t_{n}+v h\right) P_{j}(v) d v\right) P_{i}(s) d s\right]\left(\varepsilon_{C G}^{n}\right)_{j} \\
& +h \sum_{l=0}^{n-1} \sum_{j=0}^{m}\left[\int_{0}^{1}\left(\int_{0}^{1} K\left(t_{n}+s h, t_{l}+v h\right) P_{j}(v) d v\right) P_{i}(s) d s\right]\left(\varepsilon_{C G}^{l}\right)_{j} \\
& +h^{m+1}\left(\rho_{C G}^{n}\right)_{i}+\sum_{l=0}^{n-1} h^{m+2}\left(\rho_{C G}^{l}\right)_{i},
\end{aligned}
$$

where

$$
\begin{aligned}
\left(\rho_{C G}^{n}\right)_{i}:= & h \int_{0}^{1}\left(\int_{0}^{s} K\left(t_{n}+s h, t_{n}+v h\right) R_{m+1, n}(v) d v\right) P_{i}(s) d s \\
& -\int_{0}^{1} R_{m+1, n}(s) P_{i}(s) d s \\
\left(\rho_{C G}^{(n, l)}\right)_{i}:= & \int_{0}^{1}\left(\int_{0}^{1} K\left(t_{n}+s h, t_{l}+v h\right) R_{m+1, l}(v) d v\right) P_{i}(s) d s .
\end{aligned}
$$

\section{Denote}

$$
\begin{aligned}
& \varepsilon_{C G}^{n}:=\left(\left(\varepsilon_{C G}^{n}\right)_{0}, \ldots,\left(\varepsilon_{C G}^{n}\right)_{m}\right)^{T}, \\
& \rho_{C G}^{n}:=\left(\left(\rho_{C G}^{n}\right)_{0}, \ldots,\left(\rho_{C G}^{n}\right)_{m}\right)^{T}, \\
& \boldsymbol{\rho}_{C G}^{(n, l)}:=\left(\left(\rho_{C G}^{(n, l)}\right)_{0}, \ldots,\left(\rho_{C G}^{(n, l)}\right)_{m}\right)^{T} .
\end{aligned}
$$

Therefore, by the continuity, (3.20) and (3.21), we have

$$
\begin{aligned}
& \left(\begin{array}{c}
\left(P_{0}(0), \ldots, P_{m}(0)\right) \\
\left(\mathbf{A}_{C G}^{n}-h \mathbf{B}_{C G}^{n}\right)
\end{array}\right) \varepsilon_{C G}^{n} \\
= & \left(\begin{array}{c}
\left(P_{0}(1), \ldots, P_{m}(1)\right) \\
\mathbf{0}_{m \times(m+1)}
\end{array}\right) \varepsilon_{C G}^{n-1}+h \sum_{l=0}^{n-1}\left(\begin{array}{c}
\mathbf{0}_{1 \times(m+1)} \\
\mathbf{B}_{C G}^{(n, l)}
\end{array}\right) \varepsilon_{C G}^{l} \\
& \quad+h^{m+1}\left(\begin{array}{c}
R_{m+1, n-1}(1)-R_{m+1, n}(0) \\
\boldsymbol{\rho}_{C G}^{n}
\end{array}\right)+h^{m+2} \sum_{l=0}^{n-1}\left(\begin{array}{c}
0 \\
\boldsymbol{\rho}_{C G}^{(n, l)}
\end{array}\right),
\end{aligned}
$$


so

$$
\begin{aligned}
& \left(\begin{array}{c}
\left(P_{0}(0), \ldots, P_{m}(0)\right) \\
\left(\mathbf{A}_{C G}-h \mathbf{B}_{C G}^{n}\right)
\end{array}\right) \varepsilon_{C G}^{n}-\left(\begin{array}{c}
\left(P_{0}(0), \ldots, P_{m}(0)\right) \\
\left(\mathbf{A}_{C G}-h \mathbf{B}_{C G}^{n-1}\right)
\end{array}\right) \varepsilon_{C G}^{n-1} \\
= & \left(\begin{array}{c}
\left(P_{0}(1), \ldots, P_{m}(1)\right) \\
\mathbf{0}_{m \times(m+1)}
\end{array}\right) \varepsilon_{C G}^{n-1}-\left(\begin{array}{c}
\left(P_{0}(1), \ldots, P_{m}(1)\right) \\
\mathbf{0}_{m \times(m+1)}
\end{array}\right) \varepsilon_{C G}^{n-2} \\
& +h \sum_{l=0}^{n-1}\left(\begin{array}{c}
\mathbf{0}_{1 \times(m+1)} \\
\mathbf{B}_{C G}^{(n, l)}
\end{array}\right) \varepsilon_{C G}^{l}-h \sum_{l=0}^{n-2}\left(\begin{array}{c}
\mathbf{0}_{1 \times(m+1)} \\
\mathbf{B}_{C G}^{(n-1, l)}
\end{array}\right) \varepsilon_{C G}^{l}+h^{m+1} \mathbf{r}_{C G}^{n},
\end{aligned}
$$

where

$$
\begin{aligned}
\mathbf{r}_{C G}^{n}:= & \left(\begin{array}{c}
R_{m+1, n-1}(1)-R_{m+1, n}(0) \\
\rho_{C G}^{n}
\end{array}\right)-\left(\begin{array}{c}
R_{m+1, n-2}(1)-R_{m+1, n-1}(0) \\
\rho_{C G}^{n-1}
\end{array}\right) \\
& +h \sum_{l=0}^{n-1}\left(\begin{array}{c}
0 \\
\rho_{C G}^{(n, l)}
\end{array}\right)-h \sum_{l=0}^{n-2}\left(\begin{array}{c}
0 \\
\rho_{C G}^{(n-1, l)}
\end{array}\right) .
\end{aligned}
$$

Hence

$$
\begin{aligned}
& \left(\begin{array}{c}
\left(P_{0}(0), \ldots, P_{m}(0)\right) \\
\left(\mathbf{A}_{C G}-h \mathbf{B}_{C G}^{n}\right)
\end{array}\right) \varepsilon_{C G}^{n} \\
= & {\left.\left[\begin{array}{c}
\left(P_{0}(0), \ldots, P_{m}(0)\right) \\
\left(\mathbf{A}_{C G}-h \mathbf{B}_{C G}^{n-1}\right)
\end{array}\right)+\left(\begin{array}{c}
\left(P_{0}(1), \ldots, P_{m}(1)\right) \\
\mathbf{0}_{m \times(m+1)}
\end{array}\right)+h\left(\begin{array}{c}
\mathbf{0}_{1 \times(m+1)} \\
\mathbf{B}_{C G}^{(n, n-1)}
\end{array}\right)\right] \varepsilon_{C G}^{n-1} } \\
& -\left(\begin{array}{c}
\left(P_{0}(1), \ldots, P_{m}(1)\right) \\
\mathbf{0}_{m \times(m+1)}
\end{array}\right) \varepsilon_{C G}^{n-2}+h \sum_{l=0}^{n-2}\left(\begin{array}{c}
\mathbf{0}_{1 \times(m+1)} \\
\mathbf{B}_{C G}^{(n, l)}-\mathbf{B}_{C G}^{(n-1, l)}
\end{array}\right) \boldsymbol{\varepsilon}_{C G}^{l}+h^{m+1} \mathbf{r}_{C G}^{n} .
\end{aligned}
$$

By the properties of Legendre polynomials and (3.16),

$$
\begin{aligned}
\mathbf{M}_{C G} & :=\left(\begin{array}{c}
\left(P_{0}(0), \ldots, P_{m}(0)\right) \\
\mathbf{A}_{C G}
\end{array}\right)^{-1}\left(\begin{array}{c}
\left(P_{0}(1), \ldots, P_{m}(1)\right) \\
\mathbf{0}_{m \times(m+1)}
\end{array}\right) \\
& =\left(\begin{array}{ccccc}
0 & 1 & 0 & \ldots & 0 \\
0 & 0 & 3 & \ldots & 0 \\
\vdots & \vdots & \vdots & \vdots & \vdots \\
0 & 0 & 0 & \ldots & 2 m-1 \\
(-1)^{m} & (-1)^{m-1} & (-1)^{m-2} 3 & \ldots & 2 m-1
\end{array}\right)\left(\begin{array}{cccc}
1 & 1 & \ldots & 1 \\
0 & 0 & \ldots & 0 \\
\vdots & \vdots & \vdots & \vdots \\
0 & 0 & \ldots & 0
\end{array}\right) \\
& =\left(\begin{array}{cccc}
0 & 0 & \ldots & 0 \\
0 & 0 & \ldots & 0 \\
\vdots & \vdots & \vdots & \vdots \\
(-1)^{m} & (-1)^{m} & \ldots & (-1)^{m}
\end{array}\right),
\end{aligned}
$$

so

$$
\varepsilon_{C G}^{n}=\left(\mathbf{I}_{m+1}+\mathbf{M}_{C G}+\mathcal{O}(h)\right) \varepsilon_{C G}^{n-1}-\left(\mathbf{M}_{C G}+\mathcal{O}(h)\right) \varepsilon_{C G}^{n-2}
$$




$$
\begin{aligned}
& +\left(\left(\overline{\mathbf{A}}_{C G}\right)^{-1}+\mathcal{O}(h)\right) h \sum_{l=0}^{n-2}\left(\begin{array}{c}
\mathbf{0}_{1 \times(m+1)} \\
\mathbf{B}_{C G}^{(n, l)}-\mathbf{B}_{C G}^{(n-1, l)}
\end{array}\right) \varepsilon_{C G}^{l} \\
& +h^{m+1}\left(\left(\overline{\mathbf{A}}_{C G}\right)^{-1}+\mathcal{O}(h)\right) \mathbf{r}_{C G}^{n} .
\end{aligned}
$$

Therefore,

$$
\begin{aligned}
& \left(\begin{array}{c}
\varepsilon_{C G}^{n} \\
\varepsilon_{C G}^{n-1}
\end{array}\right) \\
= & {\left.\left[\begin{array}{cc}
\mathbf{I}_{m+1}+\mathbf{M}_{C G} & -\mathbf{M}_{C G} \\
\mathbf{I}_{m+1} & \mathbf{0}_{m+1}
\end{array}\right)+\mathcal{O}(h)\right]\left(\begin{array}{c}
\varepsilon_{C G}^{n-1} \\
\varepsilon_{C G}^{n-2}
\end{array}\right) } \\
& +h \sum_{l=0}^{n-2}\left(\begin{array}{c}
\left(\left(\overline{\mathbf{A}}_{C G}\right)^{-1}+\mathcal{O}(h)\right)\left(\begin{array}{c}
\mathbf{0}_{1 \times(m+1)} \\
\mathbf{B}_{C G}^{(n, l)}-\mathbf{B}_{C G}^{(n-1, l)}
\end{array}\right) \\
\mathbf{0}_{(m+1) \times(m+1)} \\
\mathbf{0}_{(m+1) \times(m+1)}
\end{array}\right)\left(\begin{array}{c}
\mathbf{0}_{C G}^{l} \\
\varepsilon_{C G}^{l-1}
\end{array}\right) \\
& +h^{m+1}\left(\begin{array}{c}
\left(\left(\overline{\mathbf{A}}_{C G}\right)^{-1}+\mathcal{O}(h)\right) \mathbf{r}_{C G}^{n} \\
\mathbf{0}_{(m+1) \times 1}
\end{array}\right) .
\end{aligned}
$$

It is obvious that the eigenvalues of the coefficient matrix

$$
\mathbf{G}_{C G}:=\left(\begin{array}{cc}
\mathbf{I}_{m+1}+\mathbf{M}_{C G} & -\mathbf{M}_{C G} \\
\mathbf{I}_{m+1} & \mathbf{0}_{m+1}
\end{array}\right)
$$

are

$$
\underbrace{1, \ldots, 1}_{m+1},(-1)^{m}, \underbrace{0, \ldots, 0}_{m} .
$$

We divide into the following two cases:

Case I: $m$ is odd. For this case, the eigenvalue 1 of multiplicity $m+1$ has $m+1$ linearly independent eigenvectors, while to the eigenvalue 0 of multiplicity $m$ there correspond $m$ linearly independent eigenvectors. Therefore, the matrix is diagonalizable, and there exists a nonsingular matrix $\mathbf{P}_{C G}$ such that

$$
\boldsymbol{\Lambda}_{C G}:=\mathbf{P}_{C G}^{-1} \mathbf{G}_{C G} \mathbf{P}_{C G}=\operatorname{diag}(1, \ldots, 1,-1,0, \ldots, 0) .
$$

Define

$$
X_{C G}^{n}:=\left\|\mathbf{P}_{C G}^{-1}\left(\begin{array}{c}
\varepsilon_{C G}^{n} \\
\varepsilon_{C G}^{n-1}
\end{array}\right)\right\|_{\infty} .
$$

Notice that by the assumption, $u \in C^{m+2}(I)$ (see [1, Theorem 2.1.3]), so $\mathbf{B}_{C G}^{(n, l)}-$ $\mathbf{B}_{C G}^{(n-1, l)}=\mathcal{O}(h)$ and $\mathbf{r}_{C G}^{n}=\mathcal{O}(h)$. Then by (3.23),

$$
X_{C G}^{n} \leq(1+\mathcal{O}(h)) X_{C G}^{n-1}+\mathcal{O}\left(h^{2}\right) \sum_{l=0}^{n-2} X_{C G}^{l}+\mathcal{O}\left(h^{m+2}\right)
$$




$$
\begin{aligned}
\leq & (1+\mathcal{O}(h))^{2} X_{C G}^{n-2}+(1+\mathcal{O}(h)) \sum_{l=0}^{n-3} \mathcal{O}\left(h^{2}\right) X_{C G}^{l} \\
& +\sum_{l=0}^{n-2} \mathcal{O}\left(h^{2}\right) X_{C G}^{l}+[1+(1+\mathcal{O}(h))] \mathcal{O}\left(h^{m+2}\right) \\
\leq & \ldots \\
\leq & (1+\mathcal{O}(h))^{n-1} X_{C G}^{1}+\sum_{k=0}^{n-2}(1+\mathcal{O}(h))^{n-2-k} \sum_{l=0}^{k} \mathcal{O}\left(h^{2}\right) X_{C G}^{l} \\
& +\sum_{k=0}^{n-2}(1+\mathcal{O}(h))^{n-2-k} \mathcal{O}\left(h^{m+2}\right) \\
\leq & \sum_{l=0}^{n-2} \mathcal{O}(h) X_{C G}^{l}+\mathcal{O}\left(h^{m+1}\right) .
\end{aligned}
$$

The discrete Gronwall inequality (see [1, Corollay 2.1.19]) yields

$$
\varepsilon_{C G}^{n}=\mathcal{O}\left(h^{m+1}\right) .
$$

By (3.20),

$$
e_{C G}\left(t_{n}+s h\right)=\sum_{j=0}^{m} P_{j}(s)\left(\varepsilon_{C G}^{n}\right)_{j}+h^{m+1} R_{m+1, n}(s)=\mathcal{O}\left(h^{m+1}\right) .
$$

Case II: $m$ is even. In order to obtain some first insight into the convergence, we first assume that $K(t, s) \equiv 1$.

For this case, the eigenvalue 1 of multiplicity $m+2$ has $m+1$ linearly independent eigenvectors, while to the eigenvalue 0 of multiplicity $m$ there correspond $m$ linearly independent eigenvectors. Therefore, there exists a nonsingular matrix $\tilde{\mathbf{P}}_{C G}$ such that

$$
\tilde{\boldsymbol{\Lambda}}_{C G}:=\tilde{\mathbf{P}}_{C G}^{-1} \mathbf{G}_{C G} \tilde{\mathbf{P}}_{C G}=\left(\begin{array}{ccccccc}
1 & 1 & & & & & \\
& 1 & & & & & \\
& & \ddots & & & & \\
& & & 1 & & & \\
& & & 0 & & \\
& & & & \ddots & \\
& & & & & & 0
\end{array}\right) .
$$

Define

$$
\tilde{\mathbf{X}}_{C G}^{n}:=\tilde{\mathbf{P}}_{C G}^{-1}\left(\begin{array}{c}
\varepsilon_{C G}^{n} \\
\varepsilon_{C G}^{n-1}
\end{array}\right) .
$$


Noticing that now $\mathbf{B}_{C G}^{(n, l)}-\mathbf{B}_{C G}^{(n-1, l)}=0$, by (3.23) we have

$$
\begin{aligned}
\tilde{\mathbf{X}}_{C G}^{n} & =\left(\tilde{\mathbf{\Lambda}}_{C G}+\mathcal{O}(h)\right) \tilde{\mathbf{X}}_{C G}^{n-1}+\mathcal{O}\left(h^{m+2}\right) \\
& =\left(\tilde{\mathbf{\Lambda}}_{C G}+\mathcal{O}(h)\right)^{2} \tilde{\mathbf{X}}_{C G}^{n-2}+\left[\mathbf{I}_{2(m+1)}+\left(\tilde{\mathbf{\Lambda}}_{C G}+\mathcal{O}(h)\right)\right] \mathcal{O}\left(h^{m+2}\right) \\
& =\ldots \\
& =\left(\tilde{\mathbf{\Lambda}}_{C G}+\mathcal{O}(h)\right)^{n} \tilde{\mathbf{X}}_{C G}^{0}+\sum_{k=0}^{n-1}\left(\tilde{\mathbf{\Lambda}}_{C G}+\mathcal{O}(h)\right)^{k} \mathcal{O}\left(h^{m+2}\right) .
\end{aligned}
$$

It is easy to check that

$$
\tilde{\Lambda}_{C G}^{n}=\left(\begin{array}{ccccccc}
1 & n & & & & & \\
& 1 & & & & & \\
& & \ddots & & & & \\
& & & 1 & & & \\
& & & & 0 & & \\
& & & & & \ddots & \\
& & & & & & 0
\end{array}\right) .
$$

Therefore,

$$
\tilde{\mathbf{X}}_{C G}^{n}=\mathcal{O}\left(h^{m}\right),
$$

and the desired result follows from (3.20).

Now we focus on the case of non-constant but arbitrarily smooth kernel $K(t, s)$. We write the CG approximation $u_{C G}$ and the exact solution in the form

$$
\begin{aligned}
& u_{C G}\left(t_{n}+s h\right)=\sum_{j=0}^{m-1} P_{j}(s)\left(\tilde{u}_{C G}\right)_{j}^{n}+h^{m} \hat{R}_{m, n}(s), \\
& u\left(t_{n}+s h\right)=\sum_{j=0}^{m-1} P_{j}(s) \hat{u}_{j}^{n}+h^{m} \tilde{R}_{m, n}(s),
\end{aligned}
$$

where the remainder terms are bounded by

$$
\left|\hat{R}_{m, n}(s)\right| \leq \frac{\left|u_{C G}^{(m)}\left(\hat{\zeta}_{n}\right)\right|}{(m+1) !}, \quad\left|\tilde{R}_{m, n}(s)\right| \leq \frac{\left|u^{(m)}\left(\hat{\zeta}_{n}^{\prime}\right)\right|}{(m+1) !},
$$

where $\hat{\zeta}_{n}, \hat{\zeta}_{n}^{\prime} \in\left(t_{n}, t_{n+1}\right)$. Therefore,

$$
e_{C G}\left(t_{n}+s h\right)=\sum_{j=0}^{m-1} P_{j}(s)\left(\tilde{\varepsilon}_{C G}^{n}\right)_{j}+h^{m} \bar{R}_{m, n}(s),
$$

where

$$
\left(\tilde{\varepsilon}_{C G}^{n}\right)_{j}:=\hat{u}_{j}^{n}-\left(\tilde{u}_{C G}\right)_{j}^{n}, \quad \bar{R}_{m, n}(s):=\tilde{R}_{m, n}(s)-\hat{R}_{m, n}(s) .
$$


By (3.12), we have

$$
\begin{aligned}
& \int_{0}^{1} e_{C G}\left(t_{n}+s h\right) P_{i}(s) d s \\
= & h \int_{0}^{1}\left(\int_{0}^{s} K\left(t_{n}+s h, t_{n}+v h\right) e_{C G}\left(t_{n}+v h\right) d v\right) P_{i}(s) d s \\
& +h \sum_{l=0}^{n-1} \int_{0}^{1}\left(\int_{0}^{1} K\left(t_{n}+s h, t_{l}+v h\right) e_{C G}\left(t_{l}+v h\right) d v\right) P_{i}(s) d s,
\end{aligned}
$$

which together with (3.24) yield

$$
\begin{aligned}
& \int_{0}^{1}\left(\sum_{j=0}^{m-1} P_{j}(s)\left(\tilde{\varepsilon}_{C G}^{n}\right)_{j}+h^{m} \bar{R}_{m, n}(s)\right) P_{i}(s) d s \\
= & h \int_{0}^{1}\left[\int_{0}^{s} K\left(t_{n}+s h, t_{n}+v h\right)\left(\sum_{j=0}^{m-1} P_{j}(v)\left(\tilde{\varepsilon}_{C G}^{n}\right)_{j}+h^{m} \bar{R}_{m, n}(v)\right) d v\right] P_{i}(s) d s \\
& +h \sum_{l=0}^{n-1} \int_{0}^{1}\left[\int_{0}^{1} K\left(t_{n}+s h, t_{l}+v h\right)\left(\sum_{j=0}^{m-1} P_{j}(v)\left(\tilde{\varepsilon}_{C G}^{l}\right)_{j}+h^{m} \bar{R}_{m, l}(v)\right) d v\right] P_{i}(s) d s,
\end{aligned}
$$

i.e.,

$$
\begin{aligned}
& \sum_{j=0}^{m-1} \int_{0}^{1} P_{j}(s) P_{i}(s) d s\left(\tilde{\varepsilon}_{C G}^{n}\right)_{j} \\
= & h \sum_{j=0}^{m-1} \int_{0}^{1}\left(\int_{0}^{s} K\left(t_{n}+s h, t_{n}+v h\right) P_{j}(v) d v\right) P_{i}(s) d s\left(\tilde{\varepsilon}_{C G}^{n}\right)_{j} \\
& +h \sum_{l=0}^{n-1} \sum_{j=0}^{m-1} \int_{0}^{1}\left(\int_{0}^{1} K\left(t_{n}+s h, t_{l}+v h\right) P_{j}(v) d v\right) P_{i}(s) d s\left(\tilde{\varepsilon}_{C G}^{l}\right)_{j}+h^{m}\left(\tilde{r}_{C G}^{n}\right)_{i},
\end{aligned}
$$

where

$$
\begin{aligned}
\left(\tilde{r}_{C G}^{n}\right)_{i}:= & -\int_{0}^{1} \bar{R}_{m, n}(s) P_{i}(s) d s+h \int_{0}^{1}\left(\int_{0}^{s} K\left(t_{n}+s h, t_{n}+v h\right) \bar{R}_{m, n}(v) d v\right) P_{i}(s) d s \\
& +h \sum_{l=0}^{n-1} \int_{0}^{1}\left(\int_{0}^{1} K\left(t_{n}+s h, t_{l}+v h\right) \bar{R}_{m, l}(v) d v\right) P_{i}(s) d s .
\end{aligned}
$$

Denote

$$
\tilde{\varepsilon}_{C G}^{n}:=\left(\left(\tilde{\varepsilon}_{C G}^{n}\right)_{0}, \ldots,\left(\tilde{\varepsilon}_{C G}^{n}\right)_{m-1}\right)^{T}, \quad \tilde{\mathbf{r}}_{C G}^{n}:=\left(\left(\tilde{r}_{C G}^{n}\right)_{0}, \ldots,\left(\tilde{r}_{C G}^{n}\right)_{m-1}\right)^{T} .
$$


Then

$$
\left(\mathbf{A}_{D G}^{n}-h \mathbf{B}_{D G}^{n}\right) \tilde{\varepsilon}_{C G}^{n}=h \sum_{l=0}^{n-1} \mathbf{B}_{D G}^{(n, l)} \tilde{\varepsilon}_{C G}^{l}+h^{m} \tilde{\mathbf{r}}_{C G}^{n} .
$$

The following proof is similar to that of the convergence for DG methods in Theorem 2.5 .

Remark 3.2. By Theorems 3.3 and 3.5, we see that both the convergence orders of the CC and CG methods are $m+1$, while the convergence of the CC method depends on the choice of collocation parameters, but the convergence of the CG method depends on the parity of $m$.

Remark 3.3. Comparing Theorem 3.5 with the results obtained in [3], we observe that the CG solution of the second-kind VIE (1.1) has a similar convergence as the DG solution of the first-kind VIE (1.3): the convergence depends on the parity of $m$. In detail, if $m$ is even, the convergence order is $m$ for the CG solution of the second-kind (1.1), while the convergence order is $m-1$ for the DG solution of the first-kind (1.1); if $m$ is odd, both of the convergence orders are raised by 1 .

\section{Numerical experiments}

In this section, we present two numerical examples to illustrate the foregoing convergence results. We choose $m=1,2,3$. For DC (QDG), CC (QCG), FDC (FDG) and FCC (FCG) methods, and we use $c_{1}=0.1,0.49,0.5,0.8,1$ respectively for $m=1$; for $m=2$ we use the Gauss collocation parameters, $c_{1}=(3-\sqrt{3}) / 6, c_{2}=(3+\sqrt{3}) / 6$; the Radau IIA collocation parameters, $c_{1}=1 / 3, c_{2}=1$; and three sets of arbitrary collocation parameters, $c_{1}=1 / 4, c_{2}=1 ; c_{1}=1 / 4, c_{2}=5 / 6 ; c_{1}=1 / 6, c_{2}=1 / 2$, respectively; for $m=3$ we use the Gauss collocation parameter, $c_{1}=(5-\sqrt{15}) / 10$, $c_{2}=1 / 2, c_{3}=(5+\sqrt{15}) / 10$; the Radau IIA collocation parameters, $c_{1}=(4-\sqrt{6}) / 10$, $c_{2}=(4+\sqrt{6}) / 10, c_{3}=1$; and three sets of arbitrary collocation parameters, $c_{1}=1 / 3$, $c_{2}=1 / 2, c_{3}=1 ; c_{1}=1 / 3, c_{2}=1 / 2, c_{3}=8 / 9 ; c_{1}=1 / 9, c_{2}=1 / 3, c_{3}=1 / 2$, respectively.

Example 4.1. We take $K(t, s) \equiv 1, g(t)=2 e^{-t}-1$. It is easy to check that the exact solution $u(t)=e^{-t}$.

In Tables 1-8, we list the maximum values of the absolute errors for DC (QDG), CC (QCG), DG and CG methods at the mesh points for Example 4.1. From these tables, we observe that the numerical results agree with our theoretical analysis, except for Radau IIA points in Tables 2, 3 and 6, where we observe the superconvergence phenomena (for DC methods with Radau IIA points in Tables 2 and 3, see [1, Corollary 2.2.8]; for CC methods with Radau IIA points in Table 6 , it can be proved in a similar way as the proof of [1, Corollary 2.2.8]). 
Table 1: The errors of $u_{D C} \in S_{m-1}^{(-1)}\left(I_{h}\right)$ for Example 4.1 with $m=1$.

\begin{tabular}{||c|c|c|c|c|c||}
\hline$N$ & $c_{1}=0.1$ & $c_{1}=0.49$ & $c_{1}=0.5$ & $c_{1}=0.8$ & $c_{1}=1$ \\
\hline $2^{9}$ & $1.7559 \mathrm{e}-03$ & $9.9419 \mathrm{e}-04$ & $9.7466 \mathrm{e}-04$ & $5.4564 \mathrm{e}-04$ & $1.1491 \mathrm{e}-03$ \\
$2^{10}$ & $8.7843 \mathrm{e}-04$ & $4.9757 \mathrm{e}-04$ & $4.8780 \mathrm{e}-04$ & $2.7263 \mathrm{e}-04$ & $5.7420 \mathrm{e}-04$ \\
$2^{11}$ & $4.3933 \mathrm{e}-04$ & $2.4890 \mathrm{e}-04$ & $2.4402 \mathrm{e}-04$ & $1.3627 \mathrm{e}-04$ & $2.8701 \mathrm{e}-04$ \\
$2^{12}$ & $2.1970 \mathrm{e}-04$ & $1.2448 \mathrm{e}-04$ & $1.2204 \mathrm{e}-04$ & $6.8123 \mathrm{e}-05$ & $1.4348 \mathrm{e}-04$ \\
Order & 1.00 & 1.00 & 1.00 & 1.00 & 1.00 \\
\hline
\end{tabular}

Table 2: The errors of $u_{D C} \in S_{m-1}^{(-1)}\left(I_{h}\right)$ for Example 4.1 with $m=2$.

\begin{tabular}{||c|c|c|c|c|c||}
\hline$N$ & Gauss & Radau IIA & $\left(\frac{1}{4}, 1\right)$ & $\left(\frac{1}{4}, \frac{5}{6}\right)$ & $\left(\frac{1}{6}, \frac{1}{2}\right)$ \\
\hline $2^{5}$ & $7.9289 \mathrm{e}-05$ & $8.3254 \mathrm{e}-07$ & $2.3253 \mathrm{e}-05$ & $5.9447 \mathrm{e}-05$ & $1.9981 \mathrm{e}-04$ \\
$2^{6}$ & $2.0082 \mathrm{e}-05$ & $1.0392 \mathrm{e}-07$ & $5.8955 \mathrm{e}-06$ & $1.5059 \mathrm{e}-05$ & $5.0406 \mathrm{e}-05$ \\
$2^{7}$ & $5.0533 \mathrm{e}-06$ & $1.2981 \mathrm{e}-08$ & $1.4841 \mathrm{e}-06$ & $3.7896 \mathrm{e}-06$ & $1.2658 \mathrm{e}-05$ \\
$2^{8}$ & $1.2674 \mathrm{e}-06$ & $1.6221 \mathrm{e}-09$ & $3.7231 \mathrm{e}-07$ & $9.5052 \mathrm{e}-07$ & $3.1717 \mathrm{e}-06$ \\
Order & 2.00 & 3.00 & 2.00 & 2.00 & 2.00 \\
\hline
\end{tabular}

Table 3: The errors of $u_{D C} \in S_{m-1}^{(-1)}\left(I_{h}\right)$ for Example 4.1 with $m=3$.

\begin{tabular}{||c|c|c|c|c|c||}
\hline$N$ & Gauss & Radau IIA & $\left(\frac{1}{3}, \frac{1}{2}, 1\right)$ & $\left(\frac{1}{3}, \frac{1}{2}, \frac{8}{9}\right)$ & $\left(\frac{1}{9}, \frac{1}{3}, \frac{1}{2}\right)$ \\
\hline $2^{3}$ & $1.4822 \mathrm{e}-05$ & $1.1005 \mathrm{e}-08$ & $1.0950 \mathrm{e}-05$ & $1.0121 \mathrm{e}-05$ & $9.0603 \mathrm{e}-05$ \\
$2^{4}$ & $1.9414 \mathrm{e}-06$ & $3.4335 \mathrm{e}-10$ & $1.3486 \mathrm{e}-06$ & $1.3860 \mathrm{e}-06$ & $1.1685 \mathrm{e}-05$ \\
$2^{5}$ & $2.4842 \mathrm{e}-07$ & $1.0718 \mathrm{e}-11$ & $1.6731 \mathrm{e}-07$ & $1.8081 \mathrm{e}-07$ & $1.4836 \mathrm{e}-06$ \\
$2^{6}$ & $3.1419 \mathrm{e}-08$ & $3.3523 \mathrm{e}-13$ & $2.0834 \mathrm{e}-08$ & $2.3074 \mathrm{e}-08$ & $1.8691 \mathrm{e}-07$ \\
Order & 2.98 & 5.00 & 3.00 & 2.97 & 2.99 \\
\hline
\end{tabular}

Table 4: The errors of $u_{C C} \in S_{m}^{(0)}\left(I_{h}\right)$ for Example 4.1 with $m=1$.

\begin{tabular}{||c|c|c|c|c|c||}
\hline & $c_{1}=0.1$ & $c_{1}=0.49$ & $c_{1}=0.5$ & $c_{1}=0.8$ & $c_{1}=1$ \\
$N$ & $\left(l_{0}(1)=-9\right)$ & $\left(l_{0}(1)=-\frac{51}{49}\right)$ & $\left(l_{0}(1)=-1\right)$ & $\left(l_{0}(1)=-\frac{1}{4}\right)$ & $\left(l_{0}(1)=0\right)$ \\
\hline $2^{8}$ & $8.0529 \mathrm{e}+237$ & $3.2424 \mathrm{e}-02$ & $3.8060 \mathrm{e}-06$ & $1.5191 \mathrm{e}-06$ & $1.4943 \mathrm{e}-06$ \\
$2^{9}$ & NaN & $2.2729 \mathrm{e}+02$ & $9.5259 \mathrm{e}-07$ & $3.8063 \mathrm{e}-07$ & $3.7359 \mathrm{e}-07$ \\
$2^{10}$ & $\mathrm{NaN}$ & $4.4675 \mathrm{e}+10$ & $2.3828 \mathrm{e}-07$ & $9.5262 \mathrm{e}-08$ & $9.3397 \mathrm{e}-08$ \\
$2^{11}$ & NaN & $6.9036 \mathrm{e}+27$ & $5.9588 \mathrm{e}-08$ & $2.3829 \mathrm{e}-08$ & $2.3349 \mathrm{e}-08$ \\
Order & - & - & 2.00 & 2.00 & 2.00 \\
\hline
\end{tabular}

Example 4.2. We take $K(t, s)=e^{t-s}, g(t)=\left(3 e^{-t}-e^{t}\right) / 2$. It is easy to check that it has the same exact solution $u(t)=e^{-t}$ as Example 4.1.

In Tables 9-14, we list the maximum values of the absolute errors for FDC (FDG) and FCC (FCG) methods at the mesh points for Example 4.2. From these tables, we observe that the numerical results agree with our theoretical analysis. We again observe the superconvergence phenomena for Radau IIA points in Tables 10, 11 and 14. 
Table 5: The errors of $u_{C C} \in S_{m}^{(0)}\left(I_{h}\right)$ for Example 4.1 with $m=2$.

\begin{tabular}{|c|c|c|c|c|c|}
\hline$N$ & $\begin{array}{c}\text { Gauss } \\
\left(l_{0}(1)=1\right)\end{array}$ & $\begin{array}{l}\text { Radau IIA } \\
\left(l_{0}(1)=0\right)\end{array}$ & $\begin{array}{c}\left(\frac{1}{4}, 1\right) \\
\left(l_{0}(1)=0\right)\end{array}$ & $\begin{array}{c}\left(\frac{1}{4}, \frac{5}{6}\right) \\
\left(l_{0}(1)=\frac{3}{5}\right)\end{array}$ & $\begin{array}{c}\left(\frac{1}{6}, \frac{1}{2}\right) \\
\left(l_{0}(1)=5\right)\end{array}$ \\
\hline $2^{5}$ & $1.4185 \mathrm{e}-05$ & $1.6383 \mathrm{e}-07$ & $2.4702 \mathrm{e}-07$ & $1.2952 \mathrm{e}-06$ & $8.7820 e+15$ \\
\hline $2^{6}$ & 3.5466e-06 & $2.0617 \mathrm{e}-08$ & $3.1006 \mathrm{e}-08$ & $1.7589 \mathrm{e}-07$ & $2.5641 \mathrm{e}+37$ \\
\hline $2^{7}$ & 8.8666e-07 & $2.5858 \mathrm{e}-09$ & 3.8836e-09 & $2.3126 \mathrm{e}-08$ & $1.7403 e+81$ \\
\hline $2^{8}$ & 2.2167e-07 & $3.2376 \mathrm{e}-10$ & 4.8595e-10 & 2.9796e-09 & $6.3981 e+169$ \\
\hline Order & 2.00 & 3.00 & 3.00 & 2.96 & - \\
\hline
\end{tabular}

Table 6: The errors of $u_{C C} \in S_{m}^{(0)}\left(I_{h}\right)$ for Example 4.1 with $m=3$.

\begin{tabular}{||c|c|c|c|c|c||}
\hline & $\begin{array}{c}\text { Gauss } \\
N\end{array}$ & $\begin{array}{c}\text { Radau IIA } \\
\left(l_{0}(1)=-1\right)\end{array}$ & $\begin{array}{c}\left(\frac{1}{3}, \frac{1}{2}, 1\right) \\
\left(l_{0}(1)=0\right)\end{array}$ & $\begin{array}{c}\left(\frac{1}{3}, \frac{1}{2}, \frac{8}{9}\right) \\
\left(l_{0}(1)=0\right)\end{array}$ & $\begin{array}{c}\left(\frac{1}{9}, \frac{1}{3}, \frac{1}{2}\right) \\
\left(l_{0}(1)=\frac{1}{4}\right)\end{array}$ \\
$\left(l_{0}(1)=16\right)$
\end{tabular}

Table 7: The errors of $u_{D G} \in S_{m-1}^{(-1)}\left(I_{h}\right)$ for Example 4.1.

\begin{tabular}{||c|c|c|c|c|c|c||}
\hline \multirow{2}{*}{$N$} & \multicolumn{2}{|c|}{$m=1$} & \multicolumn{2}{c|}{$m=2$} & \multicolumn{2}{c||}{$m=3$} \\
\cline { 2 - 7 } & $\max _{1 \leq n \leq N}\left|e\left(t_{n}\right)\right|$ & Order & $\max _{1 \leq n \leq N}\left|e\left(t_{n}\right)\right|$ & Order & $\max _{1 \leq n \leq N}\left|e\left(t_{n}\right)\right|$ & Order \\
\hline $2^{2}$ & $1.0073 \mathrm{e}-01$ & - & $4.3796 \mathrm{e}-03$ & - & $1.1098 \mathrm{e}-04$ & - \\
$2^{3}$ & $5.6223 \mathrm{e}-02$ & 0.84 & $1.1934 \mathrm{e}-03$ & 1.88 & $1.5023 \mathrm{e}-05$ & 2.89 \\
$2^{4}$ & $2.9652 \mathrm{e}-02$ & 0.92 & $3.1161 \mathrm{e}-04$ & 1.94 & $1.9545 \mathrm{e}-06$ & 2.94 \\
$2^{5}$ & $1.5222 \mathrm{e}-02$ & 0.96 & $7.9621 \mathrm{e}-05$ & 1.97 & $2.4926 \mathrm{e}-07$ & 2.97 \\
$2^{6}$ & $7.7112 \mathrm{e}-03$ & 0.98 & $2.0124 \mathrm{e}-05$ & 1.98 & $3.1472 \mathrm{e}-08$ & 2.99 \\
$2^{7}$ & $3.8809 \mathrm{e}-03$ & 0.99 & $5.0585 \mathrm{e}-06$ & 1.99 & $3.9537 \mathrm{e}-09$ & 2.99 \\
$2^{8}$ & $1.9468 \mathrm{e}-03$ & 1.00 & $1.2681 \mathrm{e}-06$ & 2.00 & $4.9546 \mathrm{e}-10$ & 3.00 \\
\hline
\end{tabular}

\section{Acknowledgments}

The author thanks Professor Hermann Brunner for his valuable comments and suggestions. They greatly improved the presentation of the results. Also, the author gratefully acknowledges Dr. Zhanwen Yang for his carefully reading the first version of the manuscript.

The work was supported by the National Nature Science Foundation of China (No. 12171122, 11771128), by the Fundamental Research Project of Shenzhen (No. JCYJ20190806143201649), Project (HIT.NSRIF.2020056), by the Natural Scientific 
Table 8: The errors of $u_{C G} \in S_{m}^{(0)}\left(I_{h}\right)$ for Example 4.1.

\begin{tabular}{||c|c|c|c|c|c|c||}
\hline \multirow{2}{*}{$N$} & \multicolumn{2}{|c|}{$m=1$} & \multicolumn{2}{c|}{$m=2$} & \multicolumn{2}{c||}{$m=3$} \\
\cline { 2 - 7 } & $\max _{1 \leq n \leq N}\left|e\left(t_{n}\right)\right|$ & Order & $\max _{1 \leq n \leq N}\left|e\left(t_{n}\right)\right|$ & Order & $\max _{1 \leq n \leq N}\left|e\left(t_{n}\right)\right|$ & Order \\
\hline $2^{2}$ & $8.7781 \mathrm{e}-03$ & - & $5.8725 \mathrm{e}-04$ & - & $4.0344 \mathrm{e}-06$ & - \\
$2^{3}$ & $2.3935 \mathrm{e}-03$ & 1.87 & $1.4677 \mathrm{e}-04$ & 2.00 & $2.7066 \mathrm{e}-07$ & 3.90 \\
$2^{4}$ & $6.2432 \mathrm{e}-04$ & 1.94 & $3.6691 \mathrm{e}-05$ & 2.00 & $1.7529 \mathrm{e}-08$ & 3.95 \\
$2^{5}$ & $1.5939 \mathrm{e}-04$ & 1.97 & $9.1726 \mathrm{e}-06$ & 2.00 & $1.1152 \mathrm{e}-09$ & 3.97 \\
$2^{6}$ & $4.0268 \mathrm{e}-05$ & 1.98 & $2.2932 \mathrm{e}-06$ & 2.00 & $7.0327 \mathrm{e}-11$ & 3.99 \\
$2^{7}$ & $1.0120 \mathrm{e}-05$ & 1.99 & $9.1726 \mathrm{e}-06$ & 2.00 & $4.4150 \mathrm{e}-12$ & 3.99 \\
$2^{8}$ & $2.5365 \mathrm{e}-06$ & 2.00 & $5.7329 \mathrm{e}-07$ & 2.00 & $2.7645 \mathrm{e}-13$ & 4.00 \\
\hline
\end{tabular}

Table 9: The errors of $u_{F D C} \in S_{m-1}^{(-1)}\left(I_{h}\right)$ for Example 4.2 with $m=1$.

\begin{tabular}{||c|c|c|c|c|c||}
\hline$N$ & $c_{1}=0.1$ & $c_{1}=0.49$ & $c_{1}=0.5$ & $c_{1}=0.8$ & $c_{1}=1$ \\
\hline $2^{9}$ & $4.2859 \mathrm{e}-03$ & $9.9420 \mathrm{e}-04$ & $9.7466 \mathrm{e}-04$ & $2.5996 \mathrm{e}-03$ & $4.5758 \mathrm{e}-03$ \\
$2^{10}$ & $2.1473 \mathrm{e}-03$ & $4.9757 \mathrm{e}-04$ & $4.8780 \mathrm{e}-04$ & $1.2996 \mathrm{e}-03$ & $2.2867 \mathrm{e}-03$ \\
$2^{11}$ & $1.0748 \mathrm{e}-03$ & $2.4890 \mathrm{e}-04$ & $2.4402 \mathrm{e}-04$ & $6.4978 \mathrm{e}-04$ & $1.1431 \mathrm{e}-03$ \\
$2^{12}$ & $5.3766 \mathrm{e}-04$ & $1.2448 \mathrm{e}-04$ & $1.2204 \mathrm{e}-04$ & $3.2488 \mathrm{e}-04$ & $5.7146 \mathrm{e}-04$ \\
Order & 1.00 & 1.00 & 1.00 & 1.00 & 1.00 \\
\hline
\end{tabular}

Table 10: The errors of $u_{F D C} \in S_{m-1}^{(-1)}\left(I_{h}\right)$ for Example 4.2 with $m=2$.

\begin{tabular}{||c|c|c|c|c|c||}
\hline$N$ & Gauss & Radau IIA & $\left(\frac{1}{4}, 1\right)$ & $\left(\frac{1}{4}, \frac{5}{6}\right)$ & $\left(\frac{1}{6}, \frac{1}{2}\right)$ \\
\hline $2^{5}$ & $7.9291 \mathrm{e}-05$ & $3.9654 \mathrm{e}-06$ & $1.8582 \mathrm{e}-04$ & $5.9454 \mathrm{e}-05$ & $4.5007 \mathrm{e}-04$ \\
$2^{6}$ & $2.0082 \mathrm{e}-05$ & $4.9586 \mathrm{e}-07$ & $4.7038 \mathrm{e}-05$ & $1.5059 \mathrm{e}-05$ & $1.1325 \mathrm{e}-04$ \\
$2^{7}$ & $5.0533 \mathrm{e}-06$ & $6.1992 \mathrm{e}-08$ & $1.1832 \mathrm{e}-05$ & $3.7896 \mathrm{e}-06$ & $2.8401 \mathrm{e}-05$ \\
$2^{8}$ & $1.2674 \mathrm{e}-06$ & $7.7495 \mathrm{e}-09$ & $2.9670 \mathrm{e}-06$ & $9.5053 \mathrm{e}-07$ & $7.1109 \mathrm{e}-06$ \\
Order & 2.00 & 3.00 & 2.00 & 2.00 & 2.00 \\
\hline
\end{tabular}

Table 11: The errors of $u_{F D C} \in S_{m-1}^{(-1)}\left(I_{h}\right)$ for Example 4.2 with $m=3$.

\begin{tabular}{||c|c|c|c|c|c||}
\hline$N$ & Gauss & Radau IIA & $\left(\frac{1}{3}, \frac{1}{2}, 1\right)$ & $\left(\frac{1}{3}, \frac{1}{2}, \frac{8}{9}\right)$ & $\left(\frac{1}{9}, \frac{1}{3}, \frac{1}{2}\right)$ \\
\hline $2^{3}$ & $1.4823 \mathrm{e}-05$ & $7.6075 \mathrm{e}-08$ & $1.6374 \mathrm{e}-04$ & $1.0643 \mathrm{e}-04$ & $3.0343 \mathrm{e}-04$ \\
$2^{4}$ & $1.9414 \mathrm{e}-06$ & $2.3845 \mathrm{e}-09$ & $2.0828 \mathrm{e}-05$ & $1.3442 \mathrm{e}-05$ & $3.8873 \mathrm{e}-05$ \\
$2^{5}$ & $2.4843 \mathrm{e}-07$ & $7.4574 \mathrm{e}-11$ & $2.6248 \mathrm{e}-06$ & $1.6876 \mathrm{e}-06$ & $4.9128 \mathrm{e}-06$ \\
$2^{6}$ & $3.1419 \mathrm{e}-08$ & $2.3323 \mathrm{e}-12$ & $3.2939 \mathrm{e}-07$ & $2.1138 \mathrm{e}-07$ & $6.1730 \mathrm{e}-07$ \\
Order & 2.98 & 5.00 & 2.99 & 3.00 & 2.99 \\
\hline
\end{tabular}

Research Innovation Foundation in Harbin Institute of Technology, and by the Research Start-Up Foundation in Harbin Institute of Technology (No. 20190019). 
Table 12: The errors of $u_{F C C} \in S_{m}^{(0)}\left(I_{h}\right)$ for Example 4.2 with $m=1$.

\begin{tabular}{||c|c|c|c|c|c||}
\hline & $c_{1}=0.1$ & $c_{1}=0.49$ & $c_{1}=0.5$ & $c_{1}=0.8$ & $c_{1}=1$ \\
$N$ & $\left(l_{0}(1)=-9\right)$ & $\left(l_{0}(1)=-\frac{51}{49}\right)$ & $\left(l_{0}(1)=-1\right)$ & $\left(l_{0}(1)=-\frac{1}{4}\right)$ & $\left(l_{0}(1)=0\right)$ \\
\hline $2^{8}$ & $8.0094 \mathrm{e}+237$ & $3.2351 \mathrm{e}-02$ & $7.7556 \mathrm{e}-06$ & $3.2485 \mathrm{e}-06$ & $1.1904 \mathrm{e}-05$ \\
$2^{9}$ & $\mathrm{NaN}$ & $2.2706 \mathrm{e}+02$ & $1.9458 \mathrm{e}-06$ & $8.1121 \mathrm{e}-07$ & $2.9760 \mathrm{e}-06$ \\
$2^{10}$ & $\mathrm{NaN}$ & $4.4652 \mathrm{e}+10$ & $4.8730 \mathrm{e}-07$ & $2.0269 \mathrm{e}-07$ & $7.4399 \mathrm{e}-07$ \\
$2^{11}$ & $\mathrm{NaN}$ & $6.9019 \mathrm{e}+27$ & $1.2193 \mathrm{e}-07$ & $5.0657 \mathrm{e}-08$ & $1.8600 \mathrm{e}-07$ \\
Order & - & - & 2.00 & 2.00 & 2.00 \\
\hline
\end{tabular}

Table 13: The errors of $u_{F C C} \in S_{m}^{(0)}\left(I_{h}\right)$ for Example 4.2 with $m=2$.

\begin{tabular}{||c|c|c|c|c|c||}
\hline & Gauss & Radau IIA & $\left(\frac{1}{4}, 1\right)$ & $\left(\frac{1}{4}, \frac{5}{6}\right)$ & $\left(\frac{1}{6}, \frac{1}{2}\right)$ \\
$N$ & $\left(l_{0}(1)=1\right)$ & $\left(l_{0}(1)=0\right)$ & $\left(l_{0}(1)=0\right)$ & $\left(l_{0}(1)=\frac{3}{5}\right)$ & $\left(l_{0}(1)=5\right)$ \\
\hline $2^{5}$ & $1.4178 \mathrm{e}-05$ & $2.6177 \mathrm{e}-06$ & $3.9421 \mathrm{e}-06$ & $1.2517 \mathrm{e}-06$ & $8.5170 \mathrm{e}+15$ \\
$2^{6}$ & $3.5461 \mathrm{e}-06$ & $3.2896 \mathrm{e}-07$ & $4.9441 \mathrm{e}-07$ & $1.7256 \mathrm{e}-07$ & $2.5256 \mathrm{e}+37$ \\
$2^{7}$ & $8.8663 \mathrm{e}-07$ & $4.1227 \mathrm{e}-08$ & $6.1901 \mathrm{e}-08$ & $2.2894 \mathrm{e}-08$ & $1.7273 \mathrm{e}+81$ \\
$2^{8}$ & $2.2166 \mathrm{e}-07$ & $5.1600 \mathrm{e}-09$ & $7.7438 \mathrm{e}-09$ & $2.9630 \mathrm{e}-09$ & $6.3743 \mathrm{e}+169$ \\
Order & 2.00 & 3.00 & 3.00 & 2.95 & - \\
\hline
\end{tabular}

Table 14: The errors of $u_{F C C} \in S_{m}^{(0)}\left(I_{h}\right)$ for Example 4.2 with $m=3$.

\begin{tabular}{||c|c|c|c|c|c||}
\hline & Gauss & Radau IIA & $\left(\frac{1}{3}, \frac{1}{2}, 1\right)$ & $\left(\frac{1}{3}, \frac{1}{2}, \frac{8}{9}\right)$ & $\left(\frac{1}{9}, \frac{1}{3}, \frac{1}{2}\right)$ \\
$N$ & $\left(l_{0}(1)=-1\right)$ & $\left(l_{0}(1)=0\right)$ & $\left(l_{0}(1)=0\right)$ & $\left(l_{0}(1)=\frac{1}{4}\right)$ & $\left(l_{0}(1)=16\right)$ \\
\hline $2^{3}$ & $4.7048 \mathrm{e}-07$ & $3.5947 \mathrm{e}-08$ & $3.0413 \mathrm{e}-06$ & $6.2312 \mathrm{e}-07$ & $4.8013 \mathrm{e}+02$ \\
$2^{4}$ & $3.0572 \mathrm{e}-08$ & $1.1518 \mathrm{e}-09$ & $1.9442 \mathrm{e}-07$ & $3.9030 \mathrm{e}-08$ & $1.4464 \mathrm{e}+11$ \\
$2^{5}$ & $1.9484 \mathrm{e}-09$ & $3.6413 \mathrm{e}-11$ & $1.2278 \mathrm{e}-08$ & $2.4392 \mathrm{e}-09$ & $1.7531 \mathrm{e}+29$ \\
$2^{6}$ & $1.2297 \mathrm{e}-10$ & $1.1453 \mathrm{e}-12$ & $7.7124 \mathrm{e}-10$ & $1.5240 \mathrm{e}-10$ & $3.8167 \mathrm{e}+66$ \\
Order & 3.99 & 4.99 & 3.99 & 4.00 & - \\
\hline
\end{tabular}

\section{References}

[1] H. Brunner, Collocation Methods for Volterra Integral and Related Functional Equations, Cambridge University Press, 2004.

[2] H. Brunner, Volterra Integral Equations: An Introduction to Theory and Applications, Cambridge University Press, 2017.

[3] H. BRunNer, P. J. DAvies, D. B. Duncan, Discontinuous Galerkin approximations for Volterra integral equations of the first kind, IMA J. Numer. Anal. 29 (2009), 856-881.

[4] H. Brunner, P. J. DAvies, D. B. Duncan, Global convergence and local superconvergence of first-kind Volterra integral equation approximations, IMA J. Numer. Anal. 32 (2012), $1117-1146$.

[5] H. BRUNNeR, Y. P. Lin, S. H. ZHANG, Higher accuracy methods for second-kind Volterra integral equations based on asymptotic expansions of iterated Galerkin methods, J. Integral Equations Appl. 10 (1998), 375-396. 
[6] H. T. CAI, Y. P. Chen, A fractional order collocation method for second kind Volterra integral equations with weakly singular kernels, J. Sci. Comput. 75 (2018), 970-992.

[7] Y. P. Chen, T. TANG, Convergence analysis of the Jacobi spectral-collocation methods for Volterra integral equations with a weakly singular kernel, Math. Comp. 79 (2010), 147167.

[8] Q. G. GuAn, R. ZHANG, Y. K. Zou, Analysis of collocation solutions for nonstandard Volterra integral equations, IMA J. Numer. Anal. 32 (2012), 1755-1785.

[9] Q. M. HuANG, M. WANG, Superconvergence of interpolated collocation solutions for weakly singular Volterra integral equations of the second kind, Comput. Appl. Math. 40 (2021), Paper No. 71, 18 pp.

[10] Q. M. HuAnG, X. X. XU, H. BRunner, Continuous Galerkin methods on quasi-geometric meshes for delay differential equaitons of pantograph type, Discrete Contin. Dynam. Systems 36 (2016), 5423-5443.

[11] W. Y. Ming, C. M. HuAnG, M. LI, Superconvergence in collocation methods for Volterra integral equations with vanishing delays, J. Comput. Appl. Math. 308 (2016), 361-378.

[12] S. S. LI, L. N. WANG, L. J. YI, An hp-version of $C^{0}$-continuous Petrov-Galerkin timestepping method for second-order Volterra integro-differential equations with weakly singular kernels, East Asian J. Appl. Math. 11 (2021), 20-42.

[13] H. LIANG, H. BRUNNER, On the convergence of collocation solutions in continuous piecewise polynomial spaces for Volterra integral equations, BIT 56 (2016), 1339-1367.

[14] H. LIANG, H. BRUNNER, The convergence of collocation solutions in continuous piecewise polynomial spaces for weakly singular Volterra integral equations, SIAM J. Numer. Anal. 57 (2019), 1875-1896.

[15] L. N. WANG, H. J. TIAN, L. J. YI, An hp-version of the discontinuous Galerkin time-stepping method for Volterra integral equations with weakly singular kernels, Appl. Numer. Math. 161 (2021), 218-232.

[16] J. Wen, A. G. XIAO, C. M. Hunang, Highly stable multistep Runge-Kutta methods for Volterra integral equations, Comput. Appl. Math. 39 (2020), Paper No. 308, 23 pp.

[17] D. XU, Second-order difference approximations for Volterra equations with the completely monotonic kernels, Numer. Algorithms 81 (2019), 1003-1041.

[18] Y. YANG, Z. Y. TANG, Mapped spectral collocation methods for Volterra integral equations with noncompact kernels, Appl. Numer. Math. 160 (2021), 166-177.

[19] Z. W. YANG, H. BRUNNER, Blow-up behavior of collocation solutions to Hammerstein-type Volterra integral equations, SIAM J. Numer. Anal. 51 (2013), 2260-2282.

[20] S. H. ZhANG, Y. P. Lin, M. RAO, Numerical solutions for second-kind Volterra integral equations by Galerkin methods, Appl. Math. 45 (2000), 19-39.

[21] J. J. ZHAO, T. LONG, Y. XU, Super implicit multistep collocation methods for weakly singular Volterra integral equations, Numer. Math. Theor. Meth. Appl. 12 (2019), 1039-1065. 